\title{
Electroweak phase diagram at finite lepton number density
}

\author{
A. Gynther* \\ Department of Physical Sciences, Theoretical Physics Division, \\ P.O. Box 64, 00014 University of Helsinki, Finland
}

\begin{abstract}
We study the thermodynamics of the electroweak theory at a finite lepton number density. The phase diagram of the theory is calculated by relating the full 4-dimensional theory to a 3-dimensional effective theory which has been previously solved using nonperturbative methods. It is seen that the critical temperature increases and the value of the Higgs boson mass at which the first order phase transition line ends decreases with increasing leptonic chemical potential.
\end{abstract}

PACS numbers: 11.10.Wx, 11.15.Ex

\section{INTRODUCTION}

The complete thermodynamic description of the electroweak theory ${ }^{1}$ depends on only five intensive variables; the temperature $T$ of the system, the strength of the external U(1) magnetic field $\boldsymbol{H}_{Y}$ and the leptonic chemical potentials $\mu_{L_{i}}$. The most studied case is that when only the temperature $T$ and the conjugated variable entropy are nonzero as it was early understood that at high temperatures the symmetry of the electroweak theory would be restored 1]. Much work was devoted to this problem using, for example, perturbative 1-loop [2, 3, 4] and 2loop [5] effective potential calculations. These only work for small scalar self-couplings or for small Higgs masses and the full solution of the problem required first a perturbative matching of the full 4-dimensional theory to an effective 3-dimensional theory 6 . The phase diagram of the effective theory was then numerically solved with lattice Monte Carlo techniques [7] (the phase diagram has been studied numerically also with the 4-dimensional theory, see [8]). The result is that the phase diagram contains a first-order line which ends in a 2nd order critical point of Ising universality class [9]. Similar techniques were then applied to solve the phase diagram when also $H_{Y}$ and the conjugate extensive variable $V B_{Y}$ were nonzero [10]. The purpose of this paper is to study the remaining case; how the phase diagram depends on finite chemical potentials related to lepton and baryon numbers and on the conjugate extensive variables, net lepton and baryon number densities.

Thermodynamical properties of the electroweak theory at nonzero lepton number density are interesting from many points of view. Theoretically, the minimal standard model describes Nature to very high accuracy and thus it is important that we know the theory completely. Especially the partition function is a fundamental concept and to know it under most general circumstances

\footnotetext{
*e-mail: antti.gynther@helsinki.fi

1 To fix the terminology, in this paper electroweak theory means the electroweak sector of the minimal standard model with all known physical parameters, essentially $G_{\mu}, m_{W}, m_{Z}$ and $m_{\text {top }}$, but parametrized with $m_{H}$.
}

is of interest. In cosmology, the neutrino degeneracy (the net neutrino number) of the universe is a poorly known number. Best limits are given by constraints from big bang nucleosynthesis and cosmic microwave background radiation which limits the degeneracy parameter $\xi_{\nu} \equiv \mu_{\nu} / T_{\nu}$ to $\xi_{\mu, \tau} \leq 2.1$ for the muon and tau neutrinos and to $\xi_{e} \leq 0.3$ for the electron neutrino where $\mu_{\nu}$ are the neutrino chemical potentials and $T_{\nu}$ is the temperature of the neutrino background 11]. If such large chemical potentials were present in the very early universe then it raises a question about how they affect the electroweak thermodynamics and especially the electroweak phase transition. It has, for example, been proposed that the presence of a large lepton number asymmetry might explain the absence of topological defects 12 as well as the observed baryon number asymmetry 13. Finally, a comparison between QCD thermodynamics and electroweak thermodynamics is interesting. QCD thermodynamics has of course attracted a lot of interest during the last years due to experiments carried out at the moment at RHIC in Brookhaven and in the future at LHC in CERN. It should be interesting to see how the properties of the QCD phase transition as a function of baryonic chemical potential and number of light flavors (strange quark mass) compare to the properties of the electroweak phase transition as a function of leptonic chemical potentials and number of light bosonic degrees of freedom (Higgs mass).

The role of finite lepton number density in the thermodynamics of the electroweak theory has been discussed already in the literature 14, 15, 16, 17, 18, 19, 20]. Those studies rely on perturbative one-loop calculations of the effective potential and the conclusion made is that the critical temperature increases with increasing chemical potentials. This can be understood in terms of BoseEinstein condensation of the Higgs field due to finite chemical potentials related to gauge charges. The fate of the $W^{ \pm}$boson condensate, predicted in [15], at high temperatures is also discussed on the same footing 17, 18]. Vector boson condensation is also discussed in 21]

Purely perturbative calculations are, however, doomed due to infrared divergences and a nonperturbative study is needed, in general. However, direct Monte Carlo studies of the full electroweak theory at high temperatures 
and finite chemical potentials are very difficult for numerous reasons. For example, the system is characterized by a multitude of scales extending from $\pi T$ (mass scale of nonzero Matsubara modes) to $g^{2} T$ (mass scale of the magnetic sector of the system). This leads to a need of large lattices in solving the properties of the system. Furthermore, chiral fermions are notoriously very hard to implement on a lattice. At finite density there is also the famous "sign" problem: the fermionic determinant is complex and thus the integration measure is not positive definite which spoils importance sampling.

We approach this problem by generalizing the successful methods of [6, 7] to finite chemical potentials. That is, we calculate the dimensional reduction of the full 4-dimensional electroweak theory at high temperatures and finite densities to a 3-dimensional effective field theory. The effective theory can then be solved using Monte Carlo methods in order to find out the phase diagram. This method is, however, by construction limited to small chemical potentials.

The paper is organized as follows. In section II we define the theories and give the matching between them. In section III we give the results for the phase diagram and in the final section, section [V] we discuss their meaning. Results for the required sum-integrals are given in the Appendix A

\section{DIMENSIONAL REDUCTION OF THE ELECTROWEAK THEORY AT FINITE CHEMICAL POTENTIALS}

In this section we describe the construction of high temperature effective field theories at finite chemical potential.

\section{A. The fundamental theory}

The electroweak theory at finite temperatures is defined by the Euclidean action

$$
\begin{aligned}
S= & \int_{0}^{\beta} \mathrm{d} \tau \int \mathrm{d}^{3} x \mathcal{L} \quad \text { with } \\
\mathcal{L}= & \left(D_{\mu} \Phi\right)^{\dagger} D_{\mu} \Phi-\nu^{2} \Phi^{\dagger} \Phi+\lambda\left(\Phi^{\dagger} \Phi\right)^{2}+\frac{1}{4} G_{\mu \nu}^{a} G_{\mu \nu}^{a} \\
& +\frac{1}{4} F_{\mu \nu} F_{\mu \nu}+\overline{\boldsymbol{l}}_{L} \not D \boldsymbol{l}_{L}+\bar{e}_{R} \not D e_{R}+\overline{\boldsymbol{q}}_{L} \not D \boldsymbol{q}_{L} \\
& +\bar{u}_{R} \not D u_{R}+\bar{d}_{R} \not D d_{R}+g_{Y}\left(\overline{\boldsymbol{q}}_{L} \tilde{\Phi} t_{R}+\bar{t}_{R} \tilde{\Phi}^{\dagger} \boldsymbol{q}_{L}\right) .
\end{aligned}
$$

Here $D_{\mu}=\partial_{\mu}+I i g A_{\mu}^{a} \tau^{a}+Y i g^{\prime} B_{\mu}$ where $I$ and $Y$ are the weak isospin and weak hypercharge of the corresponding doublet/singlet, $\boldsymbol{l}_{L}$ and $\boldsymbol{q}_{L}$ denote the left handed lepton and quark doublets and $e_{R}, u_{R}$ and $d_{R}$ denote the right handed leptons, up type quarks and down type quarks, respectively. Also, $G_{\mu \nu}^{a}=\partial_{\mu} A_{\nu}^{a}-\partial_{\nu} A_{\mu}^{a}-g \epsilon^{a b c} A_{\mu}^{b} A_{\nu}^{c}$, $F_{\mu \nu}=\partial_{\mu} B_{\nu}-\partial_{\nu} B_{\mu}$ and $\tilde{\Phi}=i \tau^{2} \Phi^{*}$. Only the top quark is taken to have a nonzero Yukawa coupling. The convention for the Euclidean gamma matrices is as given in 6]. The bosonic fields $(\phi)$ are periodic in $\tau$ while fermionic fields $(\psi)$ are anti-periodic. Thus they can be expanded in Fourier series (Matsubara modes)

$$
\begin{aligned}
\phi(\tau, \boldsymbol{x}) & =\sum_{n=-\infty}^{\infty} \phi_{n}(\boldsymbol{x}) \mathrm{e}^{i 2 n \pi T \tau}, \\
\psi(\tau, \boldsymbol{x}) & =\sum_{n=-\infty}^{\infty} \psi_{n}(\boldsymbol{x}) \mathrm{e}^{i(2 n+1) \pi T \tau} .
\end{aligned}
$$

We will employ the power-counting rules $g^{\prime 2} \sim g^{3}$, $\lambda \sim g_{Y}^{2} \sim g^{2}$. The Lagrangian is CP symmetric. Calculations are performed in Landau gauge.

As well known, the thermodynamics of any system is described by the partition function defined as trace of the density matrix

$$
\mathcal{Z}=\operatorname{Tr} \mathrm{e}^{-\beta\left(H-\mu_{k} N_{k}\right)}
$$

Here $N_{k}$ are all the conserved (global or local) charges of the system and $\mu_{k}$ are the corresponding chemical potentials. In the electroweak theory, at classical level, the lepton number currents and the baryon number current are conserved independently. However, due to the triangle anomaly, these currents are not conserved in quantum theory (for a review see, e.g. [22])

$$
\partial_{\mu} j_{\mu} \propto g^{2} \epsilon_{\alpha \beta \mu \nu} G_{\alpha \beta}^{a} G_{\mu \nu}^{a} \quad \text { for each current. }
$$

It is thus possible to form only $n_{f}$ conserved linear combinations of these currents. These are usually defined to be

$$
X_{i}=\frac{1}{n_{f}} B-L_{i}, \quad i=1 \ldots n_{f}
$$

where $n_{f}$ is the number of families, $B$ is the baryon number and $L_{i}$ are the lepton numbers for each family

$$
\begin{aligned}
B & =\frac{1}{3} \sum_{f, c} \int \mathrm{d}^{3} x \bar{q}_{c, f} \gamma_{0} q_{c, f}, \\
L_{i} & =\int \mathrm{d}^{3} x\left(\bar{e}_{i} \gamma_{0} e_{i}+\bar{\nu}_{i} \gamma_{0} a_{L} \nu_{i}\right) \\
\text { where } \quad & a_{L}=\frac{1}{2}\left(1-\gamma_{5}\right) .
\end{aligned}
$$

Here $f$ and $c$ stand for flavor and color, respectively, and $q_{c, f}$ are the quark fields. The remaining current $n_{f} B+$ $\sum_{i} L_{i}$ is not conserved.

In addition to these globally conserved charges there are locally conserved charges related to the gauge symmetries of the theory. Of the four gauge generators we can choose two mutually commuting ones for which it is possible to assign chemical potentials. One must be the hypercharge and as the other one it is convenient to 
choose the third component of the isospin. The corresponding currents are

$$
\begin{aligned}
j_{\mu}^{Y}= & \frac{1}{2} \sum_{\text {fam. }}\left(\frac{1}{3} \overline{\boldsymbol{q}}_{L} \gamma_{\mu} \boldsymbol{q}_{L}+\frac{4}{3} \bar{u}_{R} \gamma_{\mu} u_{R}-\frac{2}{3} \bar{d}_{R} \gamma_{\mu} d_{R}\right. \\
& \left.-\overline{\boldsymbol{l}}_{L} \gamma_{\mu} \boldsymbol{l}_{L}-2 \bar{e}_{R} \gamma_{\mu} e_{R}\right)-\frac{i}{2}\left(\left(D_{\mu} \Phi\right)^{\dagger} \Phi-\Phi^{\dagger} D_{\mu} \Phi\right), \\
j_{\mu}^{3}= & \frac{1}{2} \sum_{\text {fam. }}\left(\overline{\boldsymbol{q}}_{L} \gamma_{\mu} \tau^{3} \boldsymbol{q}_{L}+\overline{\boldsymbol{l}}_{L} \gamma_{\mu} \tau^{3} \boldsymbol{l}_{L}\right) \\
& -\frac{i}{2}\left(\left(D_{\mu} \Phi\right)^{\dagger} \tau^{3} \Phi-\Phi^{\dagger} \tau^{3} D_{\mu} \Phi\right)-\epsilon^{3 b c} A^{\nu, b} G_{\mu \nu}^{c} .
\end{aligned}
$$

The sums run over the families. Chemical potentials related to gauge charges cannot, however, be chosen freely. In thermal equilibrium the system must be neutral with respect to gauge charges. This requirement fixes the values of these chemical potentials, which are then functions of temperature and chemical potentials related to global charges. This can be seen explicitly below.

Taking all the conserved currents into account, the partition function is given by the path integral [18]

$$
\begin{aligned}
\mathcal{Z} & =\operatorname{Tr} \exp \left(-\beta\left(H-\mu_{i} X_{i}-\mu_{Y} Q_{Y}-\mu_{T^{3}} Q_{T^{3}}\right)\right)(9) \\
& =\int \mathcal{D} \varphi \exp \left[-\left(S-\int_{0}^{\beta} \mathrm{d} \tau \sum_{i=1}^{n_{f}} \mu_{i} X_{i}\right)\right] \\
& \equiv \int \mathcal{D} \varphi \exp \left[-S+\int_{0}^{\beta} \mathrm{d} \tau\left(\mu_{B} B+\sum_{i=1}^{n_{f}} \mu_{L_{i}} L_{i}\right)\right]
\end{aligned}
$$

where $\varphi$ denotes the set of all the fields. Here we have defined ( $\tilde{\varphi}$ excludes $B_{0}$ and $A_{0}^{3}$ )

$$
\begin{aligned}
\mu_{B} & \equiv \frac{1}{n_{f}} \sum_{i=1}^{n_{f}} \mu_{i}, \quad \mu_{L_{i}} \equiv-\mu_{i}, \quad \text { and } \\
S & =S\left[\tilde{\varphi}, B_{0}+\frac{i \mu_{Y}}{g^{\prime}}, A_{0}^{3}+\frac{i \mu_{T^{3}}}{g}\right]
\end{aligned}
$$

with $S$ containing also the gauge fixing and ghost terms. We see that the constraint $n_{f} \mu_{B}+\sum_{i} \mu_{L_{i}}=0$ is satisfied. We also note from Eqs. (10) and (12) that after the integration over the conjugated momentum fields is done (going from Eq. (9) to Eq. (10)), the chemical potentials related to the gauge charges enter the path integral the same way as the static modes of the temporal components of the corresponding gauge fields. Thus, we can interpret them as acting as backgrounds for the gauge fields. Therefore, writing $B_{0} \rightarrow B_{0}+\left\langle B_{0}\right\rangle, A_{0}^{3} \rightarrow A_{0}^{3}+\left\langle A_{0}^{3}\right\rangle$ and requiring stationarity of free energy with respect to expectation values $\left\langle B_{0}\right\rangle$ and $\left\langle A_{0}^{3}\right\rangle$ (condition for thermal equilibrium) is equivalent to requiring neutrality with respect to corresponding gauge charges:

$$
0=\frac{\partial \ln \mathcal{Z}}{\partial\left\langle B_{0}\right\rangle}=-i g^{\prime} \frac{\partial \ln \mathcal{Z}}{\partial \mu_{Y}}=-\frac{i g^{\prime} Q_{Y}}{T}
$$

and similarly for $\left\langle A_{0}^{3}\right\rangle$. It is then convenient to redefine $B_{0}$ and $A_{0}^{3}$ in Eqs. (10) and (12) as

$$
g^{\prime} B_{0} \rightarrow g^{\prime} B_{0}-i \mu_{Y}, \quad g A_{0}^{3} \rightarrow g A_{0}^{3}-i \mu_{T^{3}} .
$$

This way the action in Eq. (12) becomes the standard electroweak action for these redefined fields and $\mu_{Y}$ and $\mu_{T^{3}}$ are not explicit in the path integral anymore. The chemical potentials can be recovered as expectation values for the redefined $B_{0}$ and $A_{0}^{3}$ at equilibrium ${ }^{2}$

$$
\left\langle B_{0}\right\rangle=\frac{i \mu_{Y}}{g^{\prime}}, \quad\left\langle A_{0}^{3}\right\rangle=\frac{i \mu_{T^{3}}}{g} .
$$

This explicitly shows that these chemical potentials cannot be chosen freely, but are fixed by requiring that the system be in thermal equilibrium.

\section{B. Dimensional reduction at finite $\mu$}

Due to infrared divergences which arise when integrating over the bosonic zero modes $\phi_{0}(\boldsymbol{x})$ (static modes in $\tau$ ), the path integral (10) cannot be reliably evaluated within perturbation theory. The reason for this is that these modes are light when the temperature is much larger than any other mass scale in the theory and therefore the high temperature expansion parameter $g^{2} T / E$ is large for them. All the other modes (nonstatic in $\tau$ ), $\phi_{n \neq 0}(\boldsymbol{x})$ and $\psi_{n}(\boldsymbol{x})$, are on the other hand always very massive, $m \sim \pi T$ and can therefore be integrated out perturbatively, as can also the static modes with $|\boldsymbol{p}|>g T$. We are then led to a very natural idea of formulating a three dimensional effective field theory for the static modes $\phi_{0}(\boldsymbol{x})[\underline{6}, 23$. This effective theory is defined to be the most general theory for the static modes respecting the required symmetries. It reproduces the Green's functions for these static modes to a controllable accuracy.

Finite fermion number density affects dimensional reduction in two ways. First, the renormalization of the fields and parameters as the heavy modes are integrated out changes when compared to the case $\mu=0$. Second, the symmetries of the fundamental four dimensional theory are reduced which gives rise to terms in the effective theories absent at $\mu=0$. More precisely, the introduction of chemical potentials to the theory leads to terms in the path integral which break $\mathrm{C}$ but preserve $\mathrm{P}$ and $\mathrm{T}$ thus making the theory, in addition to being $\mathrm{C}$ and $\mathrm{P}$ breaking, also $\mathrm{CP}$ and CPT breaking. The effective theories may therefore contain terms which break $\mathrm{CP}$ and CPT and which do not appear at $\mu=0$. Such terms must nevertheless still preserve 3 -dimensional gauge- and rotational invariance as well as $\mathrm{T}$ invariance.

The first effective theory is obtained after integrating out the nonzero Matsubara modes. The resulting effective theory is a 3-dimensional $\mathrm{SU}(2) \times \mathrm{U}(1)$ gauge field theory with a fundamental scalar doublet (Higgs)

\footnotetext{
2 To be precise, this requires that the expectation values of the original $B_{0}$ and $A_{0}^{3}$ vanish in equilibrium. This certainly is the case when the system is neutral.
} 


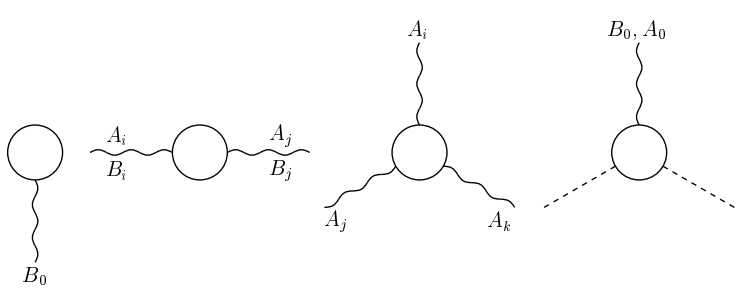

FIG. 1: Diagrams leading to new terms in the effective theories. Solid lines correspond to fermions, wavy lines to gauge fields and dashed lines to scalars.

and four adjoint scalars corresponding to the temporal components of the gauge fields in the fundamental 4-dimensional theory. The dimensionally lowest order $\mathrm{CP}$ and $\mathrm{CPT}$ violating terms arising from the finite chemical potentials in this theory are (in 3-dimensions $\left.[\varphi]=\mathrm{GeV}^{1 / 2}\right)$

$$
\begin{aligned}
\operatorname{dim}=\mathrm{GeV}^{\frac{1}{2}}: & i B_{0}, \\
\operatorname{dim}=\mathrm{GeV}^{\frac{3}{2}}: & i B_{0}^{3}, \quad i \Phi^{\dagger} A_{0}^{a} \tau^{a} \Phi, \quad i \Phi^{\dagger} B_{0} \Phi, \quad i B_{0} A_{0}^{a} A_{0}^{a}, \\
\operatorname{dim}=\mathrm{GeV}^{2}: & \epsilon_{i j k} B_{i} F_{j k}, \\
& \epsilon_{i j k}\left(A_{i}^{a} G_{j k}^{a}-\frac{i}{3} g \epsilon^{a b c} A_{i}^{a} A_{j}^{b} A_{k}^{c}\right) .
\end{aligned}
$$

which arise from diagrams given in Fig. 1] The factors of $i$ are chosen in such a way that these operators are $\mathrm{T}$ invariant (with $\mathrm{T}$ transformations adopted from the 4dimensional theory). The effective theory does not contain the terms $\operatorname{Tr} A_{0}^{a} \tau^{a}$ and $\operatorname{Tr}\left(A_{0}^{a} \tau^{a}\right)^{3}$ since these vanish identically due to the properties of the $\mathrm{SU}(2)$ generators. The coefficient of the term $B_{0}^{3}$ is of the order $g^{\prime 3} \sim g^{9 / 2}$ and when the matching of the Green's functions is done to order $g^{4}$ it can be neglected.

The possibility that there are terms linear in the ad- joint scalars in the action of the effective theory is noteworthy. Such terms induce condensates of the corresponding fields in equilibrium. As already discussed, such condensates for the adjoint scalars are equivalent to nonzero chemical potentials for the gauge charges. Thus the emergence of linear terms in the effective theory takes care of neutrality of the system with respect to gauge charges.

The last two terms of Eq. (16), the so called ChernSimons terms, are interesting. There of course is a vast literature on the physics induced by them (for a review, see 24]). That such terms appear in chiral gauge field theories when fermions are integrated out was first observed in [25]. However, in the present study we observe that the coefficients of these terms in the effective theories vanish due to the nonconservation of $B+L$ $\left(n_{f} \mu_{B}+\sum_{i} \mu_{L_{i}}=0\right)$. Thus they do not play any role in them. The role of those terms at smaller temperatures, where they may be important, has been discussed in [26].

The second effective theory is obtained after further integrating out the adjoint scalars, the zero components of the gauge fields. The resulting theory is a 3 -dimensional $\mathrm{SU}(2) \times \mathrm{U}(1)+$ Higgs gauge field theory. The form of the theory is fully determined by the gauge invariance. There cannot, therefore, be any terms in the theory that would be absent at $\mu=0$ as far as symmetries are considered (apart from the Chern-Simons terms, which nevertheless, as stated above, turn out to be absent). Finite chemical potentials show up only in the mapping of the parameters of this theory to the physical variables.

\section{Integration over the superheavy modes}

The first effective theory in its most general form is defined by the Lagrangian

$$
\begin{aligned}
\mathcal{L}_{1}= & \frac{1}{4} G_{i j}^{a} G_{i j}^{a}+\frac{1}{4} F_{i j} F_{i j}+\left(D_{i} \Phi\right)^{\dagger} D_{i} \Phi+m_{3}^{2} \Phi^{\dagger} \Phi+\lambda_{3}\left(\Phi^{\dagger} \Phi\right)^{2}+\frac{1}{2}\left(D_{i} A_{0}^{a}\right)^{2}+\frac{1}{2} m_{D}^{2} A_{0}^{a} A_{0}^{a} \\
& +\frac{1}{4} \lambda_{A} A_{0}^{a} A_{0}^{a} A_{0}^{b} A_{0}^{b}+\frac{1}{2}\left(\partial_{i} B_{0}\right)^{2}+\frac{1}{2} m_{D}^{\prime 2} B_{0}^{2}+h_{3} \Phi^{\dagger} \Phi A_{0}^{a} A_{0}^{a}+h_{3}^{\prime} \Phi^{\dagger} \Phi B_{0}^{2}+\frac{1}{2} g_{3} g_{3}^{\prime} B_{0} \Phi^{\dagger} A_{0}^{a} \tau^{a} \Phi \\
& +\alpha \epsilon_{i j k}\left(A_{i}^{a} G_{j k}^{a}-\frac{i}{3} g_{3} \epsilon^{a b c} A_{i}^{a} A_{j}^{b} A_{k}^{c}\right)+\alpha^{\prime} \epsilon_{i j k} B_{i} F_{j k}+\kappa_{1} B_{0}+\rho \Phi^{\dagger} A_{0}^{a} \tau^{a} \Phi+\rho^{\prime} \Phi^{\dagger} \Phi B_{0}+\rho_{G} B_{0} A_{0}^{a} A_{0}^{a},
\end{aligned}
$$

where $D_{i} A_{0}^{a}=\partial_{i} A_{0}^{a}-g_{3} \epsilon^{a b c} A_{i}^{b} A_{0}^{c}$. The parameters of this theory are to be matched to those of the 4-dimensional theory up to order $g^{4}$. The factors of $i$ are included in the coefficients $\kappa_{1}, \rho, \rho^{\prime}$ and $\rho_{G}$.

In general, theories are related by matching corresponding Green's functions calculated in each theory. If the fields are renormalized as ( $\varphi$ denotes a generic field)

$$
\varphi_{3 \mathrm{D}}^{2}=\frac{1}{T} \mathcal{Z}_{\varphi} \varphi_{4 \mathrm{D}}^{2}
$$

then the $N$-point Green's functions are related by

$$
\Gamma_{3 \mathrm{D}}^{\left\{n_{i}\right\}}=\frac{1}{T} \prod_{i}\left(\frac{T}{\mathcal{Z}_{i}}\right)^{n_{i} / 2} \Gamma_{4 \mathrm{D}}^{\left\{n_{i}\right\}}, \quad \sum_{i} n_{i}=N,
$$



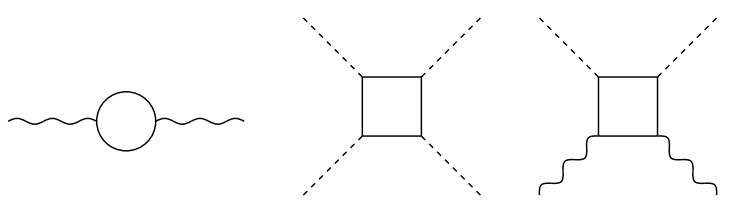

FIG. 2: The required diagrams for Debye masses and the couplings. Solid lines correspond to fermions, dashed lines to scalars and wavy lines to gauge bosons.

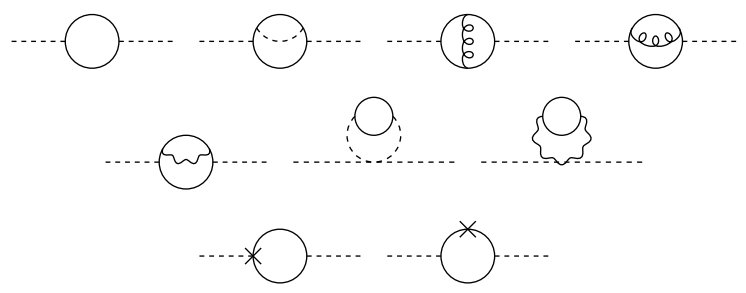

FIG. 3: The required scalar 2 point functions for the mass parameter. Solid lines correspond to fermions, dashed lines to scalars, wavy lines to electroweak gauge bosons and curly lines to gluons. Crosses denote counterterms.

where $i$ labels the different fields and $n_{i}$ is the number of times the field $i$ occurs in the Green's function. This matching has been performed for the minimal standard model at zero chemical potentials in [6]. At finite fermion number density those results are modified.

Let us denote by $\Delta \Gamma_{4 \mathrm{D}}$ and $\Delta \mathcal{Z}_{\varphi}$ the change in the 4dimensional Green's functions and field renormalizations due to finite chemical potentials, and by $\Delta \Gamma_{3 \mathrm{D}}$ the change in the 3-dimensional Green's functions due to changes in the parameters of the effective theory. We then get from Eq. (19) that

$$
\begin{aligned}
\Delta \Gamma_{3 \mathrm{D}}^{\left(\left\{n_{i}\right\}\right)}= & T^{N / 2-1}\left[\left(\prod_{i} \mathcal{Z}_{i, \mu=0}^{-n_{i} / 2}\right) \Delta \Gamma_{4 \mathrm{D}}^{\left(\left\{n_{i}\right\}\right)}\right. \\
& \left.-\left(\sum_{i} \frac{n_{i}}{2} \Delta \mathcal{Z}_{i}\right) \Gamma_{4 \mathrm{D}}^{\left(\left\{n_{i}\right\}\right)}\right]
\end{aligned}
$$

which holds when $\mathcal{Z}_{\varphi}$ are calculated up to one-loop order. This formula allows us to calculate the changes in the mapping of the parameters of the effective theory to physical variables.

\section{Changes in the coefficients of the terms present already at $\mu=0$}

The field renormalizations can be calculated from the momentum dependent part of the two-point functions. Denoting by $\mathcal{Z}_{\varphi}^{0}$ the results at zero chemical potential given in Eqs. (141), (142) and (143) of [6] we get

$$
\begin{aligned}
\mathcal{Z}_{\phi} & =\mathcal{Z}_{\phi}^{0}\left(1-\frac{3 g_{Y}^{2}}{16 \pi^{2}} \mathcal{A}\left(\frac{\mu_{B}}{3}\right)\right) \\
\mathcal{Z}_{A_{0}} & =\mathcal{Z}_{A_{0}}^{0}\left(1-\frac{g^{2}}{48 \pi^{2}}\left[9 \mathcal{A}\left(\frac{\mu_{B}}{3}\right)+\sum_{i=1}^{n_{f}} \mathcal{A}\left(\mu_{L_{i}}\right)\right]\right) \\
\mathcal{Z}_{A_{i}} & =\mathcal{Z}_{A_{i}}^{0}\left(1-\frac{g^{2}}{48 \pi^{2}}\left[9 \mathcal{A}\left(\frac{\mu_{B}}{3}\right)+\sum_{i=1}^{n_{f}} \mathcal{A}\left(\mu_{L_{i}}\right)\right]\right) .
\end{aligned}
$$

Here we have defined the function $\mathcal{A}(\mu)$ (see Appendix A]

$$
\mathcal{A}(\mu)=\psi\left(\frac{1}{2}+\frac{i \mu}{2 \pi T}\right)+\psi\left(\frac{1}{2}-\frac{i \mu}{2 \pi T}\right)+2 \gamma_{E}+2 \ln 4 .
$$

It is now straightforward to find the modifications of the renormalization of the parameters. The Debye masses get a correction coming from the diagrams in Fig. 2 These are needed only to order $g^{2}$ (as discussed in [6]) and thus the modification of the field renormalizations do not affect the Debye masses. Denoting by subscript $\mu=0$ the results from Eqs. (160) and (161) of [6] we get

$$
\begin{aligned}
& m_{D}^{2}=m_{D, \mu=0}^{2}+\frac{g^{2}}{4 \pi^{2}}\left(\mu_{B}^{2}+\sum_{i=1}^{n_{f}} \mu_{L_{i}}^{2}\right) \\
& m_{D}^{\prime 2}=m_{D, \mu=0}^{\prime 2}+\frac{g^{\prime 2}}{4 \pi^{2}}\left(\frac{11}{9} \mu_{B}^{2}+3 \sum_{i=1}^{n_{f}} \mu_{L_{i}}^{2}\right) .
\end{aligned}
$$

The coupling constants, on the other hand, are modified due to both changes in field renormalizations and changes in loop integrals. The required diagrams are shown in Fig. 2. The results are

$$
\begin{aligned}
& \lambda_{3}=\lambda_{3, \mu=0}-\frac{3 g_{Y}^{2} T}{16 \pi^{2}}\left(g_{Y}^{2}-2 \lambda\right) \mathcal{A}\left(\frac{\mu_{B}}{3}\right), \\
& h_{3}=h_{3, \mu=0}+\frac{g^{4} T}{192 \pi^{2}}\left(9 \mathcal{A}\left(\frac{\mu_{B}}{3}\right)+\sum_{i=1}^{n_{f}} \mathcal{A}\left(\mu_{L_{i}}\right)\right), \\
& g_{3}^{2}=g_{3, \mu=0}^{2}+\frac{g^{4} T}{48 \pi^{2}}\left(9 \mathcal{A}\left(\frac{\mu_{B}}{3}\right)+\sum_{i=1}^{n_{f}} \mathcal{A}\left(\mu_{L_{i}}\right)\right),(24)
\end{aligned}
$$

where $\lambda_{3, \mu=0}, h_{3, \mu=0}$ and $g_{3, \mu=0}^{2}$ are given in Eqs. (150), (147) and (146) of 6], respectively. One may note that $\lambda_{A}$, the self coupling of the adjoint scalars, does not get any corrections from the chemical potentials and thus Eq. (162) of [6] holds. Also, $h_{3}^{\prime}=g_{3}^{\prime 2} / 4$.

Last, the scalar mass parameter is calculated to two loops. One must carefully obtain all the contributions from fields renormalizations and loop integrals. The diagrams needing recalculation are shown in Fig. 3 The result is $m_{3}^{2}(\bar{\mu})=m_{3, \mu-0}^{2}(\bar{\mu})+\Delta m_{3}^{2}$ where $m_{3, \mu=0}^{2}(\bar{\mu})$ is given by Eq. (156) of [ $[6]$ and 


$$
\begin{aligned}
\Delta m_{3}^{2}= & \frac{g_{Y}^{2}(\bar{\mu})}{12 \pi^{2}} \mu_{B}^{2}-\frac{3 g_{Y}^{2}}{16 \pi^{2}}\left(\nu^{2}-\frac{\lambda T^{2}}{2}-\frac{3 g^{2} T^{2}}{16}-\frac{g_{Y}^{2} T^{2}}{4}\right) \mathcal{A}\left(\frac{\mu_{B}}{3}\right)+\frac{g_{Y}^{2} \mu_{B}^{2}}{64 \pi^{4}}\left[\frac{3}{4} g^{2} L_{b}(\bar{\mu})-g_{Y}^{2}\left(L_{f}(\bar{\mu})-\mathcal{A}\left(\frac{\mu_{B}}{3}\right)\right)\right] \\
& -\left[\left(9 g_{Y}^{4}+\frac{9}{2} g_{Y}^{2} g^{2}+16 g_{Y}^{2} g_{s}^{2}\right) \mathcal{A}\left(\frac{\mu_{B}}{3}\right)-\left(9 g_{Y}^{4}+\frac{9}{4} g_{Y}^{2} g^{2}-18 \lambda g_{Y}^{2}-16 g_{Y}^{2} g_{s}^{2}-\frac{27}{4} g^{4}\right) 16 \mathcal{B}\left(\frac{\mu_{B}}{3}\right)\right] \frac{T^{2}}{128 \pi^{2}} \\
& +\left(9 g_{Y}^{4}+\frac{9}{4} g_{Y}^{2} g^{2}-18 \lambda g_{Y}^{2}-16 g_{Y}^{2} g_{s}^{2}-\frac{27}{4} g^{4}\right) \frac{i \mu_{B} T}{48 \pi^{3}} \ln \left(\frac{\Gamma\left(\frac{1}{2}-\frac{i \mu_{B}}{6 \pi T}\right)}{\Gamma\left(\frac{1}{2}+\frac{i \mu_{B}}{6 \pi T}\right)}\right) \\
& +\left[9 g_{Y}^{2} g^{2}-6\left(3 g_{Y}^{4}-8 g_{Y}^{2} g_{s}^{2}\right) L_{b}(\bar{\mu})+9 g_{Y}^{4} L_{f}(\bar{\mu})+\left(\frac{9}{2} g_{Y}^{2} g^{2}+16 g_{Y}^{2} g_{s}^{2}\right)(4 \ln 2-1)\right. \\
& \left.+\left(9 g_{Y}^{4}+\frac{9}{4} g_{Y}^{2} g^{2}-18 \lambda g_{Y}^{2}-16 g_{Y}^{2} g_{s}^{2}-\frac{27}{4} g^{4}\right) 4 \gamma_{E}-\left(9 g_{Y}^{4}+\frac{9}{2} g_{Y}^{2} g^{2}+16 g_{Y}^{2} g_{s}^{2}\right) \mathcal{A}\left(\frac{\mu_{B}}{3}\right)\right] \frac{\mu_{B}^{2}}{1152 \pi^{4}} \\
& -\frac{3}{4} g^{4} \sum_{i=1}^{n_{f}}\left[\frac{T^{2}}{8 \pi^{2}} \mathcal{B}\left(\mu_{L_{i}}\right)+\frac{i \mu_{L_{i}} T}{16 \pi^{3}} \ln \left(\frac{\Gamma\left(\frac{1}{2}-\frac{i \mu_{L_{i}}}{2 \pi T}\right)}{\Gamma\left(\frac{1}{2}+\frac{i \mu_{L_{i}}}{2 \pi T}\right)}\right)+\frac{\mu_{L_{i}}^{2}}{32 \pi^{4}} \gamma_{E}\right] .
\end{aligned}
$$

Here the function $\mathcal{B}(\mu)$ (see Appendix $\mathbb{\AA}$ ) is defined by

$$
\begin{aligned}
\mathcal{B}(\mu)= & \zeta^{\prime}\left(-1, \frac{1}{2}+\frac{i \mu}{2 \pi T}\right)+\zeta^{\prime}\left(-1, \frac{1}{2}-\frac{i \mu}{2 \pi T}\right) \\
& -2 \zeta^{\prime}\left(-1, \frac{1}{2}\right)
\end{aligned}
$$

and the functions $L_{b}(\bar{\mu})$ and $L_{f}(\bar{\mu})$ are as defined in $[\overline{6}]$,

$$
\begin{aligned}
& L_{b}(\bar{\mu})=\ln \frac{\bar{\mu}^{2}}{T^{2}}-2 \ln 4 \pi+2 \gamma_{E} \\
& L_{f}(\bar{\mu})=\ln \frac{\bar{\mu}^{2}}{T^{2}}-2 \ln \pi+2 \gamma_{E} .
\end{aligned}
$$

Here $\bar{\mu}$ is the renormalization scale in the $\overline{\mathrm{MS}}$ scheme. Note that $\Delta m_{3}^{2}$ is independent of $\bar{\mu}$ when the running of $g_{Y}^{2}$ is taken into account.

\section{New terms}

As already pointed out, chemical potentials also induce new terms to the effective theories. These arise from the diagrams in Fig 1 The most important one of these is the term linear in $B_{0}$ which is related to the neutrality of the system. Calculating to two loops (not including contributions of the order $g^{\prime 3} \sim g^{9 / 2}$ ) we get

$$
\begin{aligned}
\kappa_{1}= & -\frac{i \pi}{3} g^{\prime} T^{5 / 2}\left[\left(1-\frac{9 g^{2}}{64 \pi^{2}}\right) \sum_{i=1}^{n_{f}} \frac{\mu_{L_{i}}}{\pi T}\left(1+\left(\frac{\mu_{L_{i}}}{\pi T}\right)^{2}\right)\right. \\
& \left.-\left(1-\frac{5 g_{Y}^{2}}{32 \pi^{2}}-\frac{9 g^{2}}{64 \pi^{2}}-\frac{g_{s}^{2}}{2 \pi^{2}}\right) \frac{\mu_{B}}{\pi T}\left(1+\frac{1}{9}\left(\frac{\mu_{B}}{\pi T}\right)^{2}\right)\right]
\end{aligned}
$$

FIG. 4: Diagrams contributing to the linear term. Solid . lines are fermions, dashed lines scalars, wavy lines electroweak gauge bosons, curly lines are gluons and external legs are $B_{0}$ (28) legs.

The corresponding diagrams are given in Fig. 4 The coefficients of the other new terms are needed only to one loop order. The result for them is

$$
\begin{aligned}
\rho & =\frac{i}{8 \pi} g g_{Y}^{2} T^{3 / 2} \frac{\mu_{B}}{\pi T}, \\
\rho^{\prime} & =-\frac{5 i}{24 \pi} g^{\prime} g_{Y}^{2} T^{3 / 2} \frac{\mu_{B}}{\pi T}, \\
\rho_{G} & =-\frac{i}{8 \pi} g^{\prime} g^{2} T^{3 / 2}\left(\frac{\mu_{B}}{\pi T}-\sum_{i=1}^{n_{f}} \frac{\mu_{L_{i}}}{\pi T}\right), \\
\alpha & =\frac{g^{2}}{32 \pi^{2}}\left(n_{f} \mu_{B}+\sum_{i=1}^{n_{f}} \mu_{L_{i}}\right)=0, \\
\alpha^{\prime} & =-\frac{g^{\prime 2}}{32 \pi^{2}}\left(n_{f} \mu_{B}+\sum_{i=1}^{n_{f}} \mu_{L_{i}}\right)=0 .
\end{aligned}
$$

These come from the diagrams in Fig. 1. As already noted, the Chern-Simons terms vanish due to the nonconservation of $B+L$ which sets $n_{f} \mu_{B}+\sum_{i} \mu_{L_{i}}=0$.

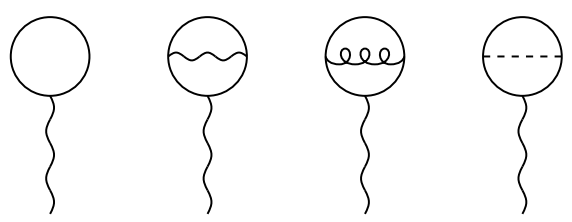




\section{One-loop effective potential}

Before integrating over the adjoint scalars $A_{0}^{a}$ and $B_{0}$ it is instructive to consider the effective potential $V_{\text {eff }}\left(\varphi,\left\langle A_{0}^{3}\right\rangle,\left\langle B_{0}\right\rangle\right)$ for the condensates $(\langle\Phi\rangle=$ $\left.1 / \sqrt{2}(0, \varphi)^{\mathrm{T}}\right)$. Although not completely reliable, $V_{\text {eff }}$ is known to give a rather good description of the phase transition at small $m_{H}$ where the scalar self coupling is small. Studies of the electroweak phase transition at finite chemical potentials using perturbatively derived effective potentials have been carried also previously [16, 17, 18].

At this point we are only interested in the qualitative effects finite chemical potentials can have. Therefore, in order to simplify the procedure, we neglect the contributions from the terms $\lambda_{A} A_{0}^{a} A_{0}^{a} A_{0}^{b} A_{0}^{b}, \quad \rho \Phi^{\dagger} A_{0}^{a} \tau^{a} \Phi, \quad \rho^{\prime} B_{0} \Phi^{\dagger} \Phi$ and $\rho_{G} B_{0} A_{0}^{a} A_{0}^{a}$.
This is motivated since $\lambda_{A} \sim g^{4}$ and $\rho_{G} \sim g^{7 / 2}$ are small and since the three point vertices $B_{0} \Phi^{\dagger} \Phi \sim \rho^{\prime}$ and $A_{0}^{a} \Phi^{\dagger} \Phi \sim \rho$ are negligible when compared to similar vertices obtained from the four point vertices $B_{0}^{2} \Phi^{\dagger} \Phi$ and $B_{0} \Phi^{\dagger} A_{0}^{a} \tau^{a} \Phi$ after annihilating a $B_{0}$ leg by the $\kappa_{1}$ vertex. Likewise, we will use $h_{3}=g_{3}^{2} / 4, h_{3}^{\prime}=g_{3}^{\prime 2} / 4$, neglecting higher order corrections, which simplifies some expressions below. This is also consistent with all the approximations above. Furthermore, we will only consider quantum fluctuations of the magnetic sector $A_{i}^{a}, B_{i}$ and of the scalars $A_{0}^{1}, A_{0}^{2}$, and treat all the condensing scalars $\Phi, B_{0}$ and $A_{0}^{3}$ only at the tree level. This approximation is adequate to show the effects of chemical potentials. A standard calculation of the one-loop effective potential gives

$$
\begin{aligned}
V_{\mathrm{eff}}\left(\varphi,\left\langle B_{0}\right\rangle,\left\langle A_{0}^{3}\right\rangle\right)= & \frac{1}{2} m_{3}^{2} \varphi^{2}+\frac{\lambda_{3}}{4} \varphi^{4}+\frac{1}{2} m_{D}^{2}\left\langle A_{0}^{3}\right\rangle^{2}+\frac{1}{2} m_{D}^{\prime 2}\left\langle B_{0}\right\rangle^{2}+\frac{1}{2}\left(h_{3}\left\langle A_{0}^{3}\right\rangle^{2}+h_{3}^{\prime}\left\langle B_{0}\right\rangle^{2}\right) \varphi^{2}-\frac{1}{4} g_{3} g_{3}^{\prime} \varphi^{2}\left\langle B_{0}\right\rangle\left\langle A_{0}^{3}\right\rangle \\
& +\kappa_{1}\left\langle B_{0}\right\rangle-\frac{1}{12 \pi}\left(\frac{3}{4} g_{3}^{3}\left(\varphi^{2}+4\left\langle A_{0}^{3}\right\rangle^{2}\right)^{3 / 2}+\frac{3}{8}\left(g_{3}^{2}+g_{3}^{\prime 2}\right)^{3 / 2} \varphi^{3}+2\left(m_{D}^{2}+h_{3} \varphi^{2}\right)^{3 / 2}\right)
\end{aligned}
$$

Requiring neutrality with respect to gauge charges enforces the conditions

$$
\frac{\partial V_{\text {eff }}}{\partial\left\langle B_{0}\right\rangle}=0 \quad \frac{\partial V_{\text {eff }}}{\partial\left\langle A_{0}^{3}\right\rangle}=0,
$$

which gives us

$$
\begin{aligned}
\left\langle B_{0}\right\rangle & =\left\langle B_{0}\right\rangle_{0}+\left\langle B_{0}\right\rangle_{1}, \quad\left\langle A_{0}^{3}\right\rangle=\left\langle A_{0}^{3}\right\rangle_{0}+\left\langle A_{0}^{3}\right\rangle_{1} \text { with } \\
\left\langle B_{0}\right\rangle_{0} & =-\frac{\kappa_{1}}{m_{D}^{\prime 2}}\left(1+\frac{h_{3}}{m_{D}^{2}} \varphi^{2}\right) \frac{1}{1+\left(\frac{h_{3}}{m_{D}^{2}}+\frac{h_{3}^{\prime}}{m_{D}^{\prime 2}}\right) \varphi^{2}}, \\
\left\langle A_{0}^{3}\right\rangle_{0} & =-\frac{\kappa_{1} g_{3} g_{3}^{\prime} \varphi^{2}}{4 m_{D}^{2} m_{D}^{\prime 2}} \frac{1}{1+\left(\frac{h_{3}}{m_{D}^{2}}+\frac{h_{3}^{\prime}}{m_{D}^{\prime 2}}\right) \varphi^{2}}, \\
\left\langle B_{0}\right\rangle_{1} & =\frac{3}{16 \pi} \frac{g_{3}^{4} g_{3}^{\prime}}{m_{D}^{2} m_{D}^{\prime 2}}\left\langle A_{0}^{3}\right\rangle_{0} \varphi^{2} \frac{\sqrt{\varphi^{2}+4\left\langle A_{0}^{3}\right\rangle_{0}^{2}}}{1+\left(\frac{h_{3}}{m_{D}^{2}}+\frac{h_{3}^{\prime}}{m_{D}^{\prime 2}}\right) \varphi^{2}}, \quad(32) \\
\left\langle A_{0}^{3}\right\rangle_{1} & =\frac{3}{4 \pi} \frac{g_{3}^{3}}{m_{D}^{2}}\left\langle A_{0}^{3}\right\rangle_{0}\left(1+\frac{h_{3}^{\prime}}{m_{D}^{\prime 2}} \varphi^{2}\right) \frac{\sqrt{\varphi^{2}+4\left\langle A_{0}^{3}\right\rangle_{0}^{2}}}{1+\left(\frac{h_{3}}{m_{D}^{2}}+\frac{h_{3}^{\prime}}{m_{D}^{\prime 2}}\right) \varphi^{2}} .
\end{aligned}
$$

Here $\left\langle B_{0}\right\rangle_{0}$ and $\left\langle A_{0}^{3}\right\rangle_{0}$ are tree level contributions to the $B_{0}$ and $A_{0}^{3}$ condensates, and $\left\langle B_{0}\right\rangle_{1}$ and $\left\langle A_{0}^{3}\right\rangle_{1}$ are cor- rections to those from the one-loop term of the effective potential. Inserting these back to the effective potential in Eq. (30) gives us then the effective potential for the Higgs expectation value

$$
\begin{aligned}
V_{\mathrm{eff}}(\varphi)= & \frac{1}{2}\left(m_{3}^{2}-\frac{1}{2 \pi} h_{3} m_{D}+\frac{h_{3}^{\prime} \kappa_{1}^{2}}{m_{D}^{\prime 4}}\right) \varphi^{2} \\
& +\frac{1}{4}\left(\lambda_{3}-\frac{1}{4 \pi} \frac{h_{3}^{2}}{m_{D}}-\frac{2 h_{3}^{\prime} \kappa_{1}^{2}}{m_{D}^{\prime 4}}\left(\frac{h_{3}}{m_{D}^{2}}+\frac{h_{3}^{\prime}}{m_{D}^{\prime 2}}\right)\right) \varphi^{4} \\
& -\frac{1}{32 \pi}\left(2 g_{3}^{3}+\left(g_{3}^{2}+g_{3}^{\prime 2}\right)^{3 / 2}\right) \varphi^{3}+\mathcal{O}\left(\varphi^{5}\right) \\
\equiv & V_{\mathrm{eff}}^{(4)}(\varphi)+\mathcal{O}\left(\varphi^{5}\right) .
\end{aligned}
$$

The error made by neglecting higher order terms in $\varphi$ is small near the phase transition as can be seen from the example in Fig. [5] In terms of the physical parameters we get $(\approx$ corresponds to only tree level matching between couplings of the 4-dimensional theory and physical variables, given for example in Eqs. (184)-(185) of [6]. The exact one-loop matching, given in Eqs. (183) and (194) of $[\underline{6}$, would not change the qualitative considerations.)

$$
\begin{aligned}
V_{\mathrm{eff}}^{(4)}(\varphi) \approx & \frac{1}{2}\left(-\frac{m_{H}^{2}}{2}+\frac{g^{2}}{16 m_{W}^{2}}\left(m_{H}^{2}+2 m_{W}^{2}+m_{Z}^{2}+2 m_{t}^{2}\right) T^{2}-\frac{16}{121} \mu^{2}+T^{2} \mathcal{O}\left(\frac{\mu^{4}}{T^{4}}, g^{2} \frac{\mu^{2}}{T^{2}}, g^{4}\right)\right) \varphi^{2} \\
& -\frac{g^{3} T^{3 / 2}}{32 \pi m_{W}^{3}}\left(2 m_{W}^{3}+m_{Z}^{3}\right) \varphi^{3}+\frac{T}{4}\left(\lambda+\frac{96}{1331} \frac{\mu^{2}}{T^{2}}+\mathcal{O}\left(\frac{\mu^{4}}{T^{4}}, g^{2} \frac{\mu^{2}}{T^{2}}, g^{4}\right)\right) \varphi^{4}
\end{aligned}
$$




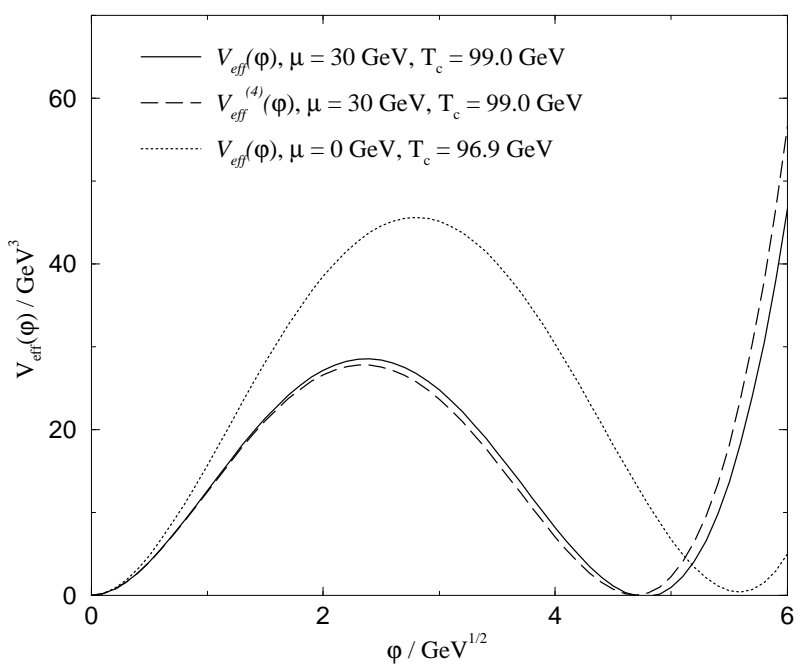

FIG. 5: The one-loop effective potentials at the phase transition point for $m_{H}=60 \mathrm{GeV}$. As can be seen, omission of the higher order terms (see Eq. (333) has only a small effect.

where we have set all the leptonic chemical potentials equal and $\mu=\mu_{L_{i}}=-\mu_{B}$ and only leading order terms in $\mu$ are kept.

This effective potential gives a qualitative picture of the effect of the chemical potentials. First, the critical temperature increases due to decrease of the Higgs mass term. Second, the scalar self-coupling increases leading to a smaller $\Delta \varphi$ at the transition and thus to a weaker transition. Also the "barrier" responsible for the first order phase transition is lower. All these effects can be seen in Fig. [5. In Fig. 6 it can be explicitly seen that finite chemical potentials tend to break the symmetry of the theory.

The leading $\mu$-induced corrections to both the scalar mass parameter and self-coupling come from the $B_{0}$ condensate. Thus, at leading order the leptonic chemical potentials change the properties of the phase transition through generating nonzero chemical potentials for the gauge charges. These couple to the Higgs field and thus change the dynamics of the Higgs field.

We can also note that the $W^{ \pm}$boson mass is reduced in the broken phase due to the $A_{0}^{3}$ condensate given in Eq. (32), ${ }^{3}$

$$
m_{W}^{2} \approx \frac{1}{4}\left(g_{3}^{2}-\frac{576}{14641} \frac{\mu^{2}}{T^{2}} \varphi^{2}\right) \varphi^{2} .
$$

If $\mu / T$ is large enough, the $W^{ \pm}$bosons may become unstable leading to a $W^{ \pm}$-condensate. We, however, restrict

\footnotetext{
3 This means that the physics behind the $W^{ \pm}$condensation is related to the nonzero chemical potentials for the gauge charges. These chemical potentials couple to the $W^{ \pm}$bosons and this condensation is nothing but Bose-Einstein condensation due to these chemical potentials.
}

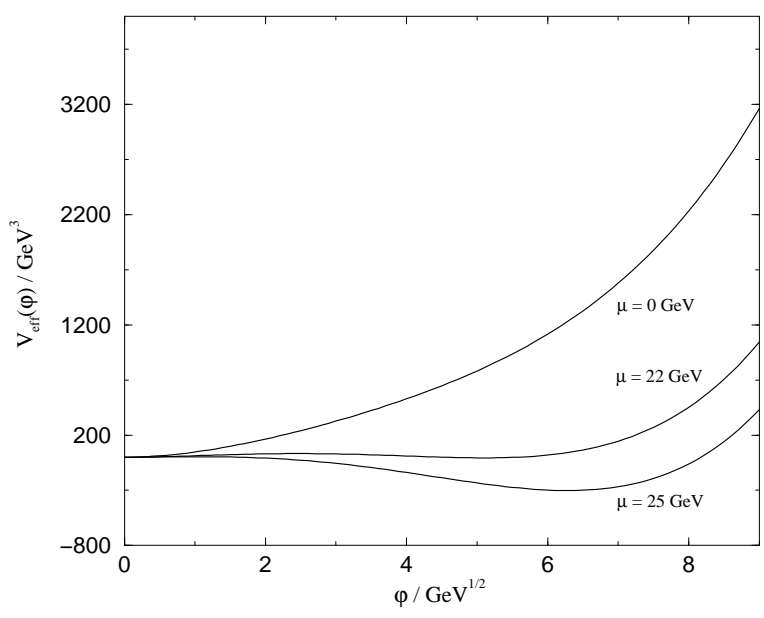

FIG. 6: The evolution of the effective potential at fixed temperature $T=98 \mathrm{GeV}$ and Higgs mass $m_{H}=60 \mathrm{GeV}$ as the chemical potential is increased.

ourselves to small chemical potentials and study phenomena near the phase transition where $\varphi^{2} \ll T$ and then the $W^{ \pm}$condensation is not relevant.

Although the perturbatively derived effective potentials serve well in giving a qualitative, and even a quantitative, picture of the phase transition, they cannot be trusted as the Higgs mass increases. This is clearly seen, for example, in predictions of the nature of the phase transition. Perturbative calculations predict a first order phase transition for all Higgs masses while nonperturbative studies show that there, in fact, is only a crossover for $m_{H} \gtrsim 72 \mathrm{GeV}$ at $\mu=0$. Since the physical Higgs mass is large, it is important not to rely on perturbative calculations.

\section{E. Integration over the adjoint scalars}

The phase transition occurs when the scalar mass parameter $m_{3}^{2}$ becomes small, $m_{3}^{2} \sim g^{4} T^{2}$. Then the adjoint scalars may be considered heavy, $m_{D}^{2} \sim g^{2} T^{2}$, and they can therefore be integrated out. The resulting effective theory is defined by the Lagrangian

$\mathcal{L}_{2}=\frac{1}{4} G_{i j}^{a} G_{i j}^{a}+\frac{1}{4} F_{i j} F_{i j}+\left(D_{i} \Phi\right)^{\dagger} D_{i} \Phi+\tilde{m}_{3}^{2} \Phi^{\dagger} \Phi+\tilde{\lambda}_{3}\left(\Phi^{\dagger} \Phi\right)^{2}$.

This reduction step $\mathcal{L}_{1} \rightarrow \mathcal{L}_{2}$ is performed in $[\underline{6}]$ in the case $\kappa_{1}=\rho=\rho^{\prime}=\rho_{G}=0$. We now generalize this to finite $\kappa_{1}, \rho, \rho^{\prime}$ and $\rho_{G}$. We use as the expansion parameter $h_{3} / m_{D}$ and the goal is to calculate the corrections up to order $\left(h_{3} / m_{D}\right)^{2} m_{D}^{2}$ for the scalar mass and to order $\left(h_{3} / m_{D}\right)^{2} m_{D}$ for the couplings in the reduction $\mathcal{L}_{1} \rightarrow \mathcal{L}_{2}$. To keep track of the contribution of different 


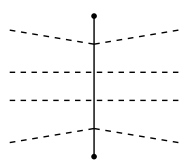

(a)

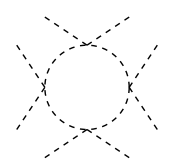

(b)

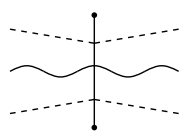

(c)
FIG. 7: (a) A tree level diagram in the theory given by $\mathcal{L}_{1}$ leading to higher order term $\left(\Phi^{\dagger} \Phi\right)^{4}$ in the last effective theory. (b) Leading order contribution to the Green's function $\left\langle\left(\Phi^{\dagger} \Phi\right)^{4}\right\rangle$ in the theory given by $\mathcal{L}_{2}$. (c) A tree level diagram in the theory given by $\mathcal{L}_{1}$ leading to higher order term $\left(\Phi^{\dagger} \Phi\right)^{2} A_{i}^{a} A_{i}^{a}$ in the last effective theory responsible for the $W^{ \pm}$condensation. Dashed lines correspond to the fundamental scalar, solid lines to adjoint scalars, wavy lines to gauge fields and dots to the $\kappa_{1}$ vertex.

terms we use the following power counting rules

$$
\begin{aligned}
& g_{3}^{2} \sim h_{3} \sim \frac{h_{3}}{m_{D}} m_{D}, \quad g_{3}^{\prime 2} \sim h_{3}^{\prime} \sim \frac{h_{3}}{m_{D}} h_{3} \sim\left(\frac{h_{3}}{m_{D}}\right)^{2} m_{D}, \\
& m_{D}^{\prime 2} \sim \frac{h_{3}}{m_{D}} m_{D}^{2}, \quad \kappa_{1} \sim\left(\frac{h_{3}}{m_{D}}\right)^{\alpha-1} m_{D}^{5 / 2}, \\
& \rho \sim\left(\frac{h_{3}}{m_{D}}\right)^{\alpha-1 / 2} m_{D}^{3 / 2}, \quad \rho^{\prime} \sim\left(\frac{h_{3}}{m_{D}}\right)^{\alpha} m_{D}^{3 / 2}, \\
& \rho_{G} \sim\left(\frac{h_{3}}{m_{D}}\right)^{2+\alpha} m_{D}^{3 / 2} .
\end{aligned}
$$

These arise from the power counting rules of the original theory supplemented by setting $\mu /(\pi T) \sim\left(h_{3} / m_{D}\right)^{\alpha}$ for some $\alpha$ where $\mu$ can be any of the chemical potentials. Due to large mass of the top quark we have also relaxed the power counting of $\rho$ and $\rho^{\prime}$ by treating $g_{Y}^{2} \sim 1$. This protects us from neglecting $\rho$ and $\rho^{\prime}$ in situations where they would be important.

It is essential to keep the chemical potentials sufficiently small in order to keep this last reduction step meaningful. Consider, for example, the set of diagrams in Fig. 17. Such diagrams would lead to terms of the form $\left(\Phi^{\dagger} \Phi\right)^{n}, n \geq 3$ in the final effective theory. If such higher order terms are not to be included in the effec- tive theory, then the contribution of those terms to the corresponding Green's functions must be sufficiently suppressed so that they can be neglected. More specifically, in the theory described by $\mathcal{L}_{2}$, the leading contribution to the Green's function $\left\langle\left(\Phi^{\dagger} \Phi\right)^{n}\right\rangle$ comes from the scalar loop in Fig. 7b and is by naive dimensional calculation of the order $\tilde{\lambda}_{3}^{n} \tilde{m}_{3}^{3-2 n} \sim\left(h_{3} / m_{D}\right)^{3-n} m_{D}^{3-n}$ near the phase transition where $\tilde{m}_{3}^{2} \sim\left(h_{3} / m_{D}\right)^{2} m_{D}^{2}$. The contribution from the graph in Fig. 7 a on the other hand is of the order of $\left(h_{3} / m_{D}\right)^{2 \alpha+n-3} m_{D}^{3-n}$. Thus, if we require that the effective theory can reproduce the Green's functions up to order $g^{3} \sim\left(h_{3} / m_{D}\right)^{3}$ and we neglect the higher order operators produced by the graphs in Fig. 7a, then we must require that

$$
\alpha \gtrsim \frac{3}{2} \quad \text { or equivalently } \quad \frac{\mu}{\pi T} \lesssim g^{\prime}
$$

This determines the powercounting rules for $\kappa_{1}, \rho, \rho^{\prime}$ and $\rho_{G}$ which are used when integrating out the adjoint scalars.

Another set of interesting tree level diagrams are those in Fig. 17. They would lead to terms of the form $\left(\Phi^{\dagger} \Phi\right)^{n} A_{i}^{a} A_{i}^{a}$ in the effective theory $\mathcal{L}_{2}$. These are interesting since it is these terms that are responsible for the $W^{ \pm}$condensation, as can, for example, be seen from Eq. (35). There the leading correction to the $W^{ \pm}$mass is $\sim \varphi^{4}$ and thus the term in the effective theory $\mathcal{L}_{2}$ that would be responsible for this correction would be $\sim\left(\Phi^{\dagger} \Phi\right)^{2} A_{i}^{a} A_{i}^{a}$. The above determined power counting rules allow us to neglect these terms but this means that the effective theory $\mathcal{L}_{2}$ cannot predict $W^{ \pm}$condensation. As already discussed, this is not a problem when we study phenomena near the phase transition and at small chemical potentials.

Since the reduction at $\kappa_{1}=\rho=\rho^{\prime}=\rho_{G}=0$ is given in [6] we now only need to take into account the contribution from the new terms. Calculating to the accuracy mentioned before, they only contribute to the scalar mass parameter (up to one-loop level) and scalar self coupling (up to tree level). Other contributions are of higher order. The required diagrams are shown in Fig. 8. The results for the parameters are

$$
\begin{aligned}
& \tilde{m}_{3}^{2}=\tilde{m}_{3,0}^{2}+\frac{h_{3}^{\prime} \kappa_{1}^{2}}{m_{D}^{\prime 4}}-\frac{\rho^{\prime} \kappa_{1}}{m_{D}^{\prime 2}}-\frac{1}{4 \pi}\left[\left(\frac{3 g_{3}^{2} g_{3}^{\prime 2}}{4 m_{D}}+\frac{4 h_{3}^{\prime 2}}{m_{D}^{\prime}}\right) \frac{\kappa_{1}^{2}}{m_{D}^{\prime 4}}-\left(\frac{3 g_{3} g_{3}^{\prime} \rho}{m_{D}}+\frac{4 h_{3}^{\prime} \rho^{\prime}}{m_{D}^{\prime}}\right) \frac{\kappa_{1}}{m_{D}^{\prime 2}}+\frac{3 \rho^{2}}{m_{D}}+\frac{\rho^{\prime 2}}{m_{D}^{\prime}}\right] \\
& \tilde{\lambda}_{3}=\tilde{\lambda}_{3,0}-\frac{1}{2}\left[\left(\frac{g_{3}^{2} g_{3}^{\prime 2}}{4 m_{D}^{2}}+\frac{4 h_{3}^{\prime 2}}{m_{D}^{\prime 2}}\right) \frac{\kappa_{1}^{2}}{m_{D}^{\prime 4}}-\left(\frac{g_{3} g_{3}^{\prime} \rho}{m_{D}^{2}}+\frac{4 h_{3}^{\prime} \rho^{\prime}}{m_{D}^{\prime 2}}\right) \frac{\kappa_{1}}{m_{D}^{\prime 2}}+\frac{\rho^{2}}{m_{D}^{2}}+\frac{\rho^{\prime 2}}{m_{D}^{\prime 2}}\right] .
\end{aligned}
$$

Here $\tilde{m}_{3,0}^{2}$ and $\tilde{\lambda}_{3,0}$ are as given in Eqs. (174) (first equality) and (169) of [6] (where they are denoted by $\bar{m}_{3}^{2}$ and $\left.\bar{\lambda}_{3}\right)$. All the other couplings of $\mathcal{L}_{2}$ are as given in [6] as functions of the parameters of $\mathcal{L}_{1}$.

The construction of the theories $\mathcal{L}_{1}$ and $\mathcal{L}_{2}$ differs in one qualitative aspect. Although neither theory is applicable at high chemical potentials, $\mu>\pi T$, there never- 


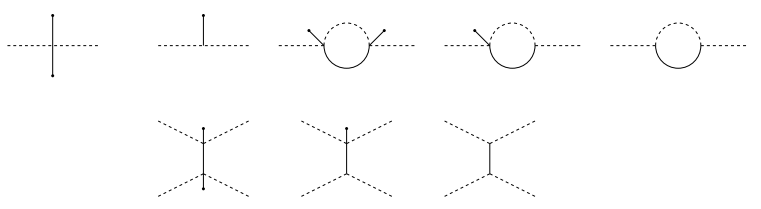

FIG. 8: Additional diagrams needed in integration over the adjoint scalars. The dashed lines correspond to the fundamental scalar, solid lines to adjoint scalars and the dot is the $\kappa_{1}$ vertex.

theless was no expansion in $\mu /(\pi T)$ in the construction of $\mathcal{L}_{1}$. That is, the matching of the parameters of $\mathcal{L}_{1}$ to those of the 4-dimensional theory is given to a certain accuracy in $g^{2}$ for arbitrary $\mu$. This is not true for the matching between $\mathcal{L}_{1}$ and $\mathcal{L}_{2}$. There it was essential to assume that the chemical potential is small. This is easy to understand since some of the couplings of $\mathcal{L}_{1}$ are directly proportional to $\mu /(\pi T)$ and thus $\mu$ must be small when $\mathcal{L}_{1}$ is studied perturbatively. We may therefore assume that $\mathcal{L}_{1}$ is applicable to somewhat higher chemical potentials than $\mathcal{L}_{2}$, up to $\mu \lesssim \pi T$. In the range $g^{\prime} \pi T \leq \mu \leq \pi T$ the dynamics of $\mathcal{L}_{1}$ may be dominated by nonperturbative effects.

\section{THE PHASE DIAGRAM}

The effective theory for the light modes is infrared divergent in perturbation theory and thus it cannot be reliably studied perturbatively. It has, however, been studied nonperturbatively by Monte Carlo studies in [7].

The theory is parametrized by four parameters. It is convenient to express three of them in a dimensionless form while leaving one of them to give the energy scale. We define

$$
x \equiv \frac{\tilde{\lambda}_{3}}{\tilde{g}_{3}^{2}}, \quad y \equiv \frac{\tilde{m}_{3}^{2}\left(\tilde{g}_{3}^{2}\right)}{\tilde{g}_{3}^{4}}, \quad z \equiv \frac{\tilde{g}_{3}^{\prime 2}}{\tilde{g}_{3}^{2}}
$$

and leave $\tilde{g}_{3}^{2}$ to give the dimensions. The value of $z$ is essentially fixed by the Weinberg mixing angle, $z \approx$ $\tan ^{2} \theta_{W} \approx 0.3$. The value of $y$ is tuned to find the phase transition at a fixed value of $x$ which determines the nature of the phase transition.

The phase diagram of the effective theory is given in Fig. 9] The continuous line is a curve fitted to the lattice results which are given in [7] and [9] for SU(2)+Higgs gauge theory. The effect of the $U(1)$ subgroup is to increase the critical $y$ slightly 7 ]. The critical line given by a perturbative calculation is given by the dashed line. As can be seen, the perturbative result gives quite a good estimate for the value of $y$ at the transition, $y=y_{c}(x)$, for small $x$. It, however, fails completely in describing the nature of the transition at high $x$. Perturbation theory predicts a first order phase transition for all $x$, while

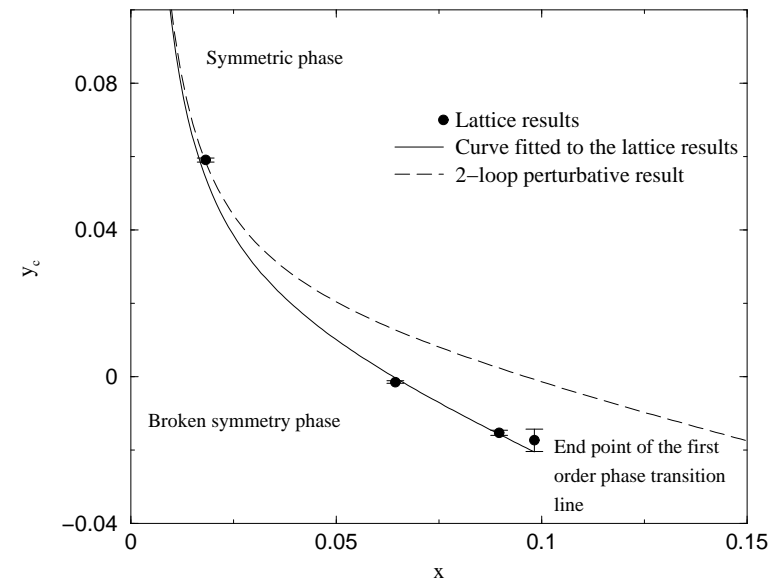

FIG. 9: The phase diagram of the effective theory. The results are from 7] and 9].
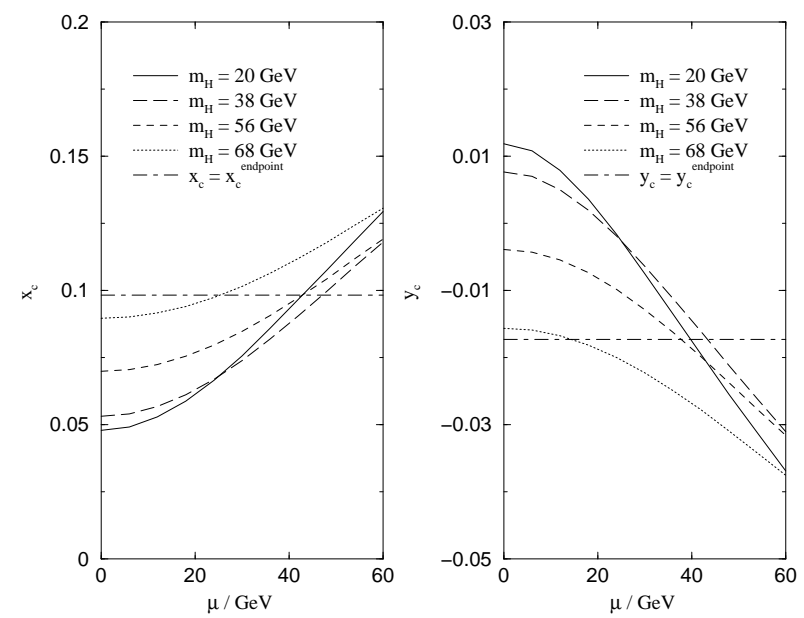

FIG. 10: Behavior of $x_{c}$ and $y_{c}$ as a function of $\mu$ for different Higgs masses. The regions above the line $x_{c}=x_{c}^{\text {endpoint }}$ and below the line $y_{c}=y_{c}^{\text {endpoint }}$ correspond to the crossover region of the theory.

Monte Carlo studies have shown that there is a first order phase transition at small $x$ but that the first order phase transition line has a second order endpoint at $x \approx 0.0983$, $y \approx-0.0173$ and for larger $x$ no phase transition is observed 7, 9]. Thus, there is no phase transition for sufficiently large Higgs masses.

The phase diagram in Fig. 9] can be expressed in terms of the physical parameters by the mapping described in section [II. For simplicity we set all the leptonic chemical potentials to be equal to each other, $\mu_{L_{i}}=\mu=-\mu_{B}$. The theory is specified by giving the physical parameters the values $G_{\mu}=1.664 \cdot 10^{-5} \mathrm{GeV}^{-2}, m_{W}=$ $80.42 \mathrm{GeV}, \quad m_{Z}=91.19 \mathrm{GeV}, m_{t}=174.3 \mathrm{GeV}$ and $\alpha_{s}\left(m_{Z}\right)=0.118$. The Higgs mass is left as a free parameter.

The exact relation between $\left(m_{H}, T, \mu\right)$ and $(x, y)$ 
is a complicated function of $\mu$. However, the essential features of the effect of the chemical potentials can be seen quite easily by just taking the leading corrections due to finite $\mu$ into account. We get

$$
\begin{aligned}
x(\mu) \approx & x(0)+\frac{1}{g^{2}} \frac{96}{1331} \frac{\mu^{2}}{T^{2}} \approx \frac{m_{H}^{2}}{8 m_{W}^{2}}+\frac{1}{g^{2}} \frac{96}{1331} \frac{\mu^{2}}{T^{2}} \\
y(\mu) \approx & y(0)-\frac{1}{g^{4}} \frac{16}{121} \frac{\mu^{2}}{T^{2}} \\
\approx & -\frac{m_{H}^{2}}{2 g^{4} T^{2}}+\frac{1}{g^{2}}\left(\frac{m_{H}^{2}}{16 m_{W}^{2}}+\frac{3}{16}+\frac{1}{16} \frac{g^{\prime 2}}{g^{2}}+\frac{1}{4} \frac{g_{Y}^{2}}{g^{2}}\right) \\
& -\frac{1}{g^{4}} \frac{16}{121} \frac{\mu^{2}}{T^{2}} .
\end{aligned}
$$

Thus the effect of finite $\mu$ is to increase $x$ and decrease $y$. Perturbatively, at tree level the phase transition occurs at $y=y_{c}=0$. The critical temperature can then be solved to be

$$
T_{0}^{2}=\frac{1}{8 \lambda+3 g^{2}+g^{2}+4 g_{Y}^{2}}\left(8 m_{H}^{2}+\frac{256}{121} \mu^{2}\right)
$$

which explicitly shows that a finite chemical potential increases the critical temperature. At one-loop order the critical line is given by $y_{c} x=1 /\left(128 \pi^{2}\right)$ and expanding this around the tree level solution $T_{c}=T_{0}$ we get for the critical temperature (to first order in $\left.\left(T_{c}-T_{0}\right) / T_{0}\right)$

$$
T_{c}=T_{0}\left(1+\frac{1}{16 \pi^{2}} \frac{g^{6}}{8 \lambda+3 g^{2}+g^{2}+4 g_{Y}^{2}} \frac{1}{\lambda+\frac{96}{1331} \frac{\mu^{2}}{T_{0}^{2}}}\right) .
$$

The factor multiplying the tree level critical temperature decreases as the chemical potentials are increased but that decrease is negligible in comparison to the simultaneous increase in $T_{0}$. Thus the critical temperature increases also at one-loop order.

The above reasoning gives a valid qualitative understanding of the behaviour of the system but to obtain quantitatively more reliable results we must map the phase diagram in Fig. 9 to $\left(T, \mu, m_{H}\right)$ using the complete results from section III Then the values of $x$ and $y$ along the phase transition line, $x_{c}=x\left(T_{c}, \mu, m_{H}\right)$ and $y_{c}=y\left(T_{c}, \mu, m_{H}\right)$, are given in Fig. [10 as functions of $\mu$. It can be observed that as the chemical potentials are increased, the subsequent increase and decrease of $x_{c}$ and $y_{c}$, respectively, are fastest at small Higgs masses. This is easy to understand since $x_{c}$ and $y_{c}$ are essentially functions of $\mu / T$. Thus, at small Higgs masses where the critical temperature is lower, increasing $\mu$ leads to a larger increase in $\mu / T_{c}$ than at large Higgs masses where the critical temperature is higher. Therefore the changes in $x_{c}$ and $y_{c}$ are also larger at smaller Higgs masses.

This has an interesting consequence. The $x_{c}(\mu)$ curves for different $m_{H}$ intersect and for sufficiently large chemical potentials the value of $x_{c}$ is, in fact, a decreasing function of the Higgs mass (at least for sufficiently small Higgs

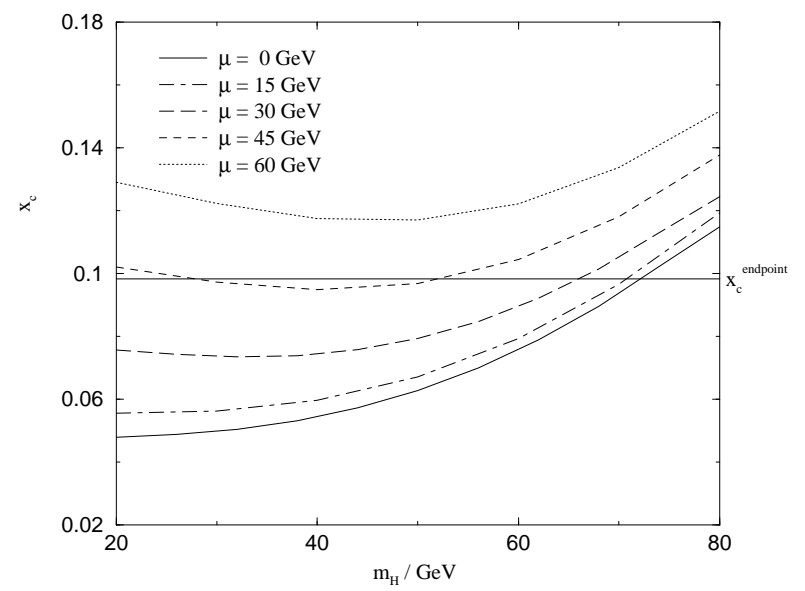

FIG. 11: The behavior of $x_{c}$ as a function of $m_{H}$.

masses). This can be seen explicitly in Fig. 11. Therefore, under these specific conditions, the phase transition appears to become stronger as the Higgs mass is increased (again, at least as long as the Higgs mass remains sufficiently small). This, however, does not seem to have any physical relevance. Dimensional reduction is not reliable at small Higgs masses where this effect is strongest. As the Higgs mass is increased also the chemical potentials must be increased in order to recover this anomalous behaviour. However, at these larger Higgs masses and chemical potentials, the value of $x_{c}$ is above the endpoint value $x_{c}^{\text {endpoint }}=0.0983$ and there is therefore no phase transition. Especially, at physical Higgs masses, $m_{H} \gtrsim 115 \mathrm{GeV}$, there is no phase transition.

The phase diagram in terms of the physical parameters is given in Figs. [12 and 13] The qualitative picture based on perturbation theory can be seen to be correct. The critical temperature increases with $\mu$. Some interesting thermodynamics can be deduced from this. According to the Clausius-Clapeyron relations

$$
\frac{d T}{d \mu}=-\frac{n_{s}-n_{b}}{s_{s}-s_{b}}
$$

where $d T / d \mu$ is measured along the phase transition line, and $n_{s, b}$ and $s_{s, b}$ are the lepton number and entropy densities in each phase, respectively. Since $d T / d \mu$ is positive, the lepton number difference between the phases is of the opposite sign than the entropy difference. Furthermore, since entropy is higher in the symmetric phase (the high temperature phase) it means that the lepton number density is higher in the broken symmetry phase.

The behaviour of the endpoint of the first order phase transition line is also of interest. For a fixed Higgs mass, a finite chemical potential strengthens the scalar selfcoupling at the transition point as seen from Fig. 10] Thus, increasing $\mu$ weakens the transition and the value of the critical Higgs mass where the first order phase transition line ends in a second order endpoint is a decreasing function of $\mu$. Especially, there cannot be a first order electroweak phase transition at physical Higgs masses in 


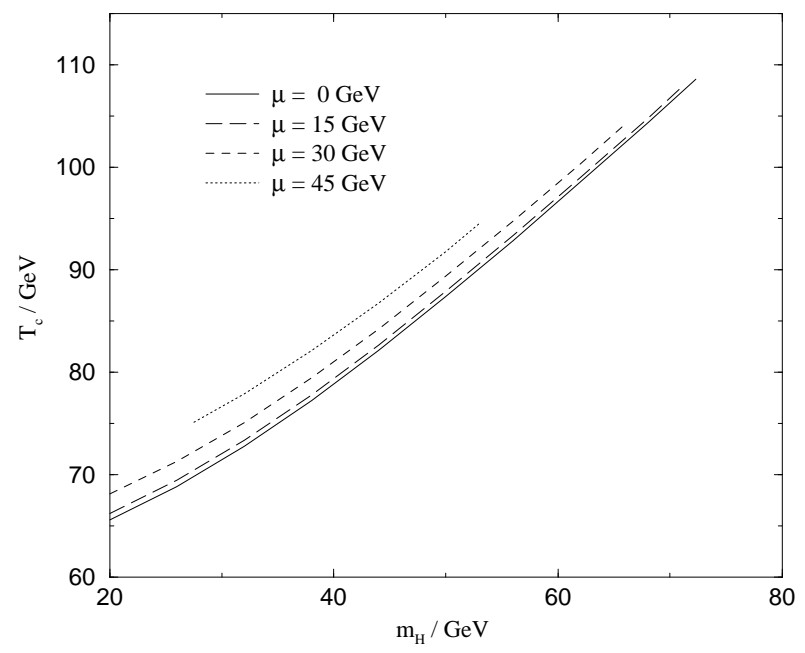

FIG. 12: The electroweak phase diagram on the $m_{H}-T$ plane.

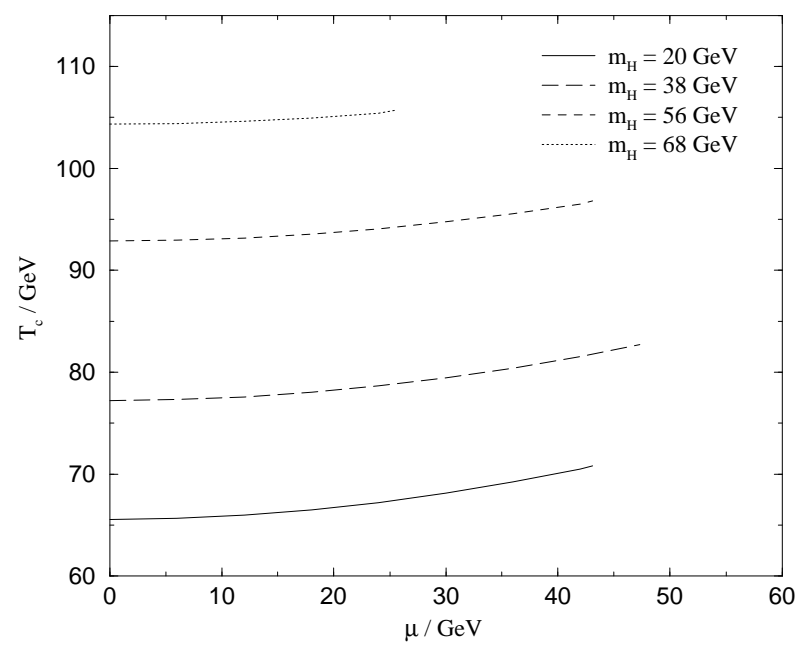

FIG. 13: The electroweak phase diagram on the $\mu-T$ plane.

the minimal standard model even when $\mu \neq 0$. Moreover, for sufficiently high chemical potential there appears to be no phase transition for any value of $m_{H}$. The location of the second order endpoint in terms of the Higgs mass and critical temperature are given in table \ for some values of the chemical potential.

\begin{tabular}{|c|c|c|}
\hline$\mu$ & $m_{H}^{\text {end point }}$ & $T_{c}^{\text {end point }}$ \\
\hline $0 \mathrm{GeV}$ & $72 \mathrm{GeV}$ & $109 \mathrm{GeV}$ \\
\hline $15 \mathrm{GeV}$ & $71 \mathrm{GeV}$ & $108 \mathrm{GeV}$ \\
\hline $30 \mathrm{GeV}$ & $66 \mathrm{GeV}$ & $104 \mathrm{GeV}$ \\
\hline $45 \mathrm{GeV}$ & $52 \mathrm{GeV}$ & $94 \mathrm{GeV}$ \\
\hline
\end{tabular}

TABLE I: The location of the endpoint of the first order phase transition line.

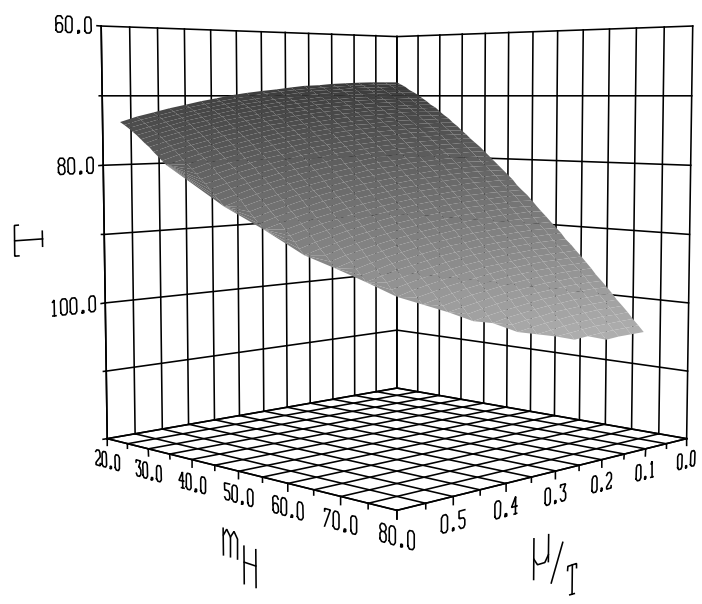

FIG. 14: The phase diagram. Note that the temperature grows downwards

\section{DISCUSSION}

In this paper we have determined quantitatively how the equilibrium phase diagram of the electroweak theory, parametrized by the Higgs mass, depends on the temperature and small leptonic chemical potentials. In particular, we have studied the change of the second order endpoint of the first order phase transition line. It is seen that the critical temperature increases and the critical value of Higgs mass where the first order phase transition line ends decreases as the chemical potentials are increased. These results are summarized in Fig. 14

It is interesting to qualitatively compare these results for the critical temperature $T=T_{c}\left(m_{H}, \mu\right)$ of the electroweak phase transition to the critical temperature $T=T_{c}\left(m_{s}, \mu_{B}\right), m_{s}=$ strange quark mass, $\mu_{B}=$ baryonic chemical potential, of the QCD phase transition. In QCD there is also a first order critical line ending in a 2nd order point: if $\mu_{B}=0$ then for $m_{s}=0\left(N_{f}=3\right)$ there is a first order phase transition while for $m_{s}=\infty$ $\left(N_{f}=2\right)$ there is only a crossover. Thus, the situation on the $m_{s}-T$ plane is like in Fig. 12. However, in contrast to Fig. 13] on the $\mu_{B}-T$ plane the critical lines for different $m_{s}$ bend downwards starting from $\mu_{B}=0$ (in Fig. 12 the $\mu_{B}>0$ curves would reside below the $\mu_{B}=0$ curve). As discussed after Eq. (44), this implies that in the QCD phase transition the entropy density and the baryon number density change in a similar way. Also, in QCD, when $m_{s}$ is large enough, there is a first order phase transition only if $\mu_{B}$ is greater than some critical value $\mu_{B, c}\left(m_{s}\right)$, again in contrast to Fig. 13$]$ which says that there is a first order electroweak phase transition only if $\mu$ is less then some critical value $\mu_{c}\left(m_{H}\right)$. 
The effective theory used in this paper to study the electroweak phase transition cannot be used at large chemical potentials. Therefore, it cannot, for example, predict the emergence (or perhaps lack of it) of the $W^{ \pm}$ condensate. The reason for this is that the driving force behind the $W^{ \pm}$condensation, Bose-Einstein condensation, would appear in this theory through higher order terms of the form $\left(\Phi^{\dagger} \Phi\right)^{n} A_{i}^{a} A_{i}^{a}, n \geq 2$. Those terms would modify the $W^{ \pm}$mass in such a way that the condensation might occur. We have, however, neglected such higher order terms from the theory which is possible if the chemical potentials are small. The $W^{ \pm}$condensation is in any case a high $\mu$ phenomenon and thus our approximation is self consistent.

\section{Acknowledgements}

The author would like to thank K. Kajantie for suggesting the topic and for numerous discussions during the work. Discussions with M. Laine and A. Vuorinen are also gratefully acknowledged. The work has been supported by the Graduate School for Particle and Nuclear Physics, GRASPANP.

\section{APPENDIX A: SUM-INTEGRALS AT FINITE $\mu$}

In this appendix we give the results for the required fermionic sum-integrals for arbitrary $\mu$. Similar integrals also appear in the context of computing QCD quark number susceptibilities [27].

\section{One-loop integrals}

At one-loop we have two types of integrals. Both of these are easily evaluated by first doing the $3-2 \epsilon$ dimen- sional momentum integration and then performing the sum using Riemann zeta functions. Denoting the sumintegrals by

$$
\sum_{p} \equiv\left(\frac{\mathrm{e}^{\gamma_{E}} \bar{\mu}^{2}}{4 \pi}\right)^{\epsilon} \sum_{n=-\infty}^{\infty} \int \frac{d^{3-2 \epsilon} p}{(2 \pi)^{3-2 \epsilon}}, \quad p_{0}=(2 n+1) \pi T-i \mu
$$

where $\bar{\mu}$ is the scale in the $\overline{\mathrm{MS}}$ scheme, we get

$$
\begin{aligned}
I_{\alpha}^{2 n} \equiv & \sum_{p_{f}} \frac{p_{0}^{2 n}}{\left(p^{2}\right)^{\alpha}} \\
= & \frac{\Gamma(\alpha-3 / 2+\epsilon)}{8 \pi^{3 / 2} \Gamma(\alpha)}\left(\mathrm{e}^{\gamma_{E}} \bar{\mu}^{2}\right)^{\epsilon} T(2 \pi T)^{2 n+3-2 \epsilon-2 \alpha} \\
& \times 2 \operatorname{Re}\left[\zeta\left(2 \alpha-2 n-3+2 \epsilon, \frac{1}{2}-\frac{i \mu}{2 \pi T}\right)\right], \\
I_{\alpha}^{2 n+1} \equiv & \oiint_{p_{f}} \frac{p_{0}^{2 n+1}}{\left(p^{2}\right)^{\alpha}} \\
= & \frac{\Gamma(\alpha-3 / 2+\epsilon)}{8 \pi^{3 / 2} \Gamma(\alpha)}\left(\mathrm{e}^{\gamma_{E}} \bar{\mu}^{2}\right)^{\epsilon} T(2 \pi T)^{2 n+4-2 \epsilon-2 \alpha} \\
& \times 2 i \operatorname{Im}\left[\zeta\left(2 \alpha-2 n-4+2 \epsilon, \frac{1}{2}-\frac{i \mu}{2 \pi T}\right)\right],
\end{aligned}
$$

where

$$
\zeta(z, q)=\sum_{n=0}^{\infty} \frac{1}{(n+q)^{z}}, \quad z>1
$$

is the generalized zeta function. Its properties are discussed below. Especially the following special cases, expanded around $\epsilon=0$, are needed:

$$
\begin{aligned}
I_{1}^{0}= & -\frac{T^{2}}{24}\left\{1+\frac{3}{\pi^{2}} \frac{\mu^{2}}{T^{2}}+\epsilon\left[\left(1+\frac{3}{\pi^{2}} \frac{\mu^{2}}{T^{2}}\right)\left(L_{f}(\bar{\mu})-2 \gamma_{E}+2-4 \ln 2\right)\right.\right. \\
& \left.\left.+24 \zeta^{\prime}\left(-1, \frac{1}{2}-\frac{i \mu}{2 \pi T}\right)+24 \zeta^{\prime}\left(-1, \frac{1}{2}+\frac{i \mu}{2 \pi T}\right)\right]\right\}+\mathcal{O}\left(\epsilon^{2}\right), \\
I_{2}^{0}= & \frac{1}{16 \pi^{2}}\left[\frac{1}{\epsilon}+L_{f}(\bar{\mu})-2 \gamma_{E}-4 \ln 2-\psi\left(\frac{1}{2}-\frac{i \mu}{2 \pi T}\right)-\psi\left(\frac{1}{2}+\frac{i \mu}{2 \pi T}\right)\right] \\
& +\mathcal{O}(\epsilon), \\
I_{1}^{1}= & \frac{i \pi T^{3}}{12} \frac{\mu}{\pi T}\left(1+\frac{\mu^{2}}{\pi^{2} T^{2}}\right)+\mathcal{O}(\epsilon), \\
I_{2}^{1}= & \frac{i T}{8 \pi} \frac{\mu}{\pi T}+\mathcal{O}(\epsilon),
\end{aligned}
$$

where the primes in zeta functions denote derivatives with respect to the first argument and $\psi(z) \equiv \partial_{z} \ln \Gamma(z)$.
The results are exact in $\mu$. Terms of the order $\epsilon$ are 
needed only for the first sum-integral.

\section{Two-loop integrals}

Most of the needed two-loop sum-integrals can be reduced to products of one-loop sum-integrals. Only the sum-integral corresponding to the "setting sun" diagram must be evaluated explicitly. In calculating it we follow the procedure outlined in [28].

The integral we are interested in is

$$
\begin{aligned}
I_{\text {sunset }} & \equiv \oint_{p_{f}, k_{b}} \frac{1}{p^{2} k^{2}(p+k)^{2}}=\oint_{k_{b}} \frac{1}{k^{2}} \Pi^{f}(k) \\
p_{0} & =(2 n+1) \pi T-i \mu, \quad k_{0}=2 m \pi T, \quad n, m \in \mathbb{Z},
\end{aligned}
$$

where we have defined the integral $\Pi^{f}(k)$ as in 28]. By going to configuration space where the propagator is given by (at $\epsilon=0)$

$$
\Delta\left(p_{0}, \boldsymbol{r}\right)=\frac{\mathrm{e}^{-\left|p_{0}\right| r}}{4 \pi r}
$$

this integral becomes

$$
\begin{aligned}
\Pi^{f}(k) & =T \sum_{\left\{p_{0}\right\}} \int \mathrm{d}^{3} r \mathrm{e}^{i \boldsymbol{k} \cdot \boldsymbol{r}} \Delta\left(p_{0}, \boldsymbol{r}\right) \Delta\left(p_{0}+k_{0}, \boldsymbol{r}\right) \\
& =\frac{T}{16 \pi^{2}} \sum_{\left\{p_{0}\right\}} \int \mathrm{d}^{3} r \frac{\mathrm{e}^{i \boldsymbol{k} \cdot \boldsymbol{r}}}{r^{2}} \mathrm{e}^{-\left|p_{0}\right| r} \mathrm{e}^{-\left|p_{0}+k_{0}\right| r} .
\end{aligned}
$$

Here we have used the notation of [28] where $\sum_{\left\{p_{0}\right\}}$ means summation over fermionic Matsubara modes.

The influence of the chemical potentials resides now in the sum

$$
\sum_{\left\{p_{0}\right\}} \mathrm{e}^{-\left|p_{0}\right| r} \mathrm{e}^{-\left|p_{0}+k_{0}\right| r}=\mathrm{e}^{-\left|k_{0}\right| r}\left(\frac{\cos (2 \mu r)}{\sinh (2 \pi T r)}+\frac{\left|k_{0}\right|}{2 \pi T}\right)
$$

and thus we get

$$
\Pi^{f}(k)=\frac{T}{16 \pi^{2}} \int \mathrm{d}^{3} r \frac{\mathrm{e}^{i \boldsymbol{k} \cdot \boldsymbol{r}}}{r^{2}}\left(\frac{\cos (2 \mu r)}{\sinh (2 \pi T r)}+\frac{\left|k_{0}\right|}{2 \pi T}\right) \mathrm{e}^{-\left|k_{0}\right| r} .
$$

From here we can proceed as in 28] by first subtracting the $T=0, \mu=0$ part (which contains the ultraviolet divergences) and then evaluating the remaining integral. The final result is

$$
\begin{aligned}
I_{\text {sunset }}= & -\frac{\mu^{2}}{64 \pi^{4}}\left(\frac{1}{\epsilon}+2 \ln \frac{\bar{\mu}^{2}}{T^{2}}-4 \ln 4 \pi+2\right) \\
& +\frac{i \mu T}{16 \pi^{3}} \ln \frac{\Gamma\left(\frac{1}{2}-\frac{i \mu}{2 \pi T}\right)}{\Gamma\left(\frac{1}{2}+\frac{i \mu}{2 \pi T}\right)}+\mathcal{O}(\epsilon) .
\end{aligned}
$$

We were unable to find whether this integral had been calculated at finite $\mu$ previously.

\section{Some properties of the required special functions}

The central function is the generalized zeta function $\zeta(z, q)$, defined in Eq. A33 for $\operatorname{Re}(z)>1$, and its derivative with respect to $z, \zeta^{\prime}(z, q)$. From the integral representation

$$
\zeta(z, q)=\frac{1}{\Gamma(z)} \int_{0}^{\infty} d t t^{z-1} \frac{\mathrm{e}^{(1-q) t}}{\mathrm{e}^{t}-1}
$$

valid at $\operatorname{Re}(z)>1, \operatorname{Re}(q)>0$, it is possible to analytically continue this function to the double complex plane $(z, q), z \neq 1$. For us it is sufficient to consider only the half plane $\operatorname{Re}(q)>0$ where we can write

$$
\begin{aligned}
\zeta(z, q)= & \frac{1}{\Gamma(z)} \sum_{n=0}^{\infty} \frac{B_{n}(q)}{\Gamma(n+1)} \frac{(-1)^{n}}{z+n-1} \\
& +\frac{1}{\Gamma(z)} \int_{1}^{\infty} \mathrm{d} t t^{z-1} \frac{\mathrm{e}^{(1-q) t}}{\mathrm{e}^{t}-1} .
\end{aligned}
$$

Here $B_{n}(q)$ are Bernoulli polynomials. This expression is well defined as long as $z \neq 1$ and $\operatorname{Re}(q)>0$ (the limit of $1 /(\Gamma(z)(z+n-1))$ is well defined at $z=0,-1,-2, \ldots)$ and thus it can be used to define the analytic continuation of $\zeta(z, q)$ to that region. For the derivative we get

$$
\begin{aligned}
\zeta^{\prime}(z, q)= & -\frac{1}{\Gamma(z)} \sum_{n=0}^{\infty} \frac{B_{n}(q)}{\Gamma(n+1)} \frac{(-1)^{n}}{(z+n-1)^{2}} \\
& +\frac{1}{\Gamma(z)} \int_{1}^{\infty} d t t^{z-1} \ln t \frac{\mathrm{e}^{(1-q) t}}{\mathrm{e}^{t}-1}-\psi(z) \zeta(z, q) .
\end{aligned}
$$

All the required special functions appearing in the integrals can be related to these functions. This is trivially true for the one-loop integrals by Eqs. (A2). The logarithm of the gamma functions present in the sunset integral Eq. A13 can on the other hand be written as $(x=\mu /(\pi T))$

$$
\ln \frac{\Gamma\left(\frac{1}{2}-\frac{i x}{2}\right)}{\Gamma\left(\frac{1}{2}+\frac{i x}{2}\right)}=\zeta^{\prime}\left(0, \frac{1}{2}-\frac{i x}{2}\right)-\zeta^{\prime}\left(0, \frac{1}{2}+\frac{i x}{2}\right) .
$$

Thus the mathematical properties of the non-polynomial part in $\mu / T$ of all the required integrals are given by the generalized zeta function.

The asymptotic behaviour of the integrals at the limits $\mu / T \rightarrow 0$ and $T / \mu \rightarrow 0$ is of interest. The small $\mu$ limit is a straightforward Taylor expansion and is not given here explicitly. The large $\mu$ limit for the required functions is most easily obtained from Stirling's approximation for the gamma function:

$$
\ln \frac{\Gamma\left(\frac{1}{2}-\frac{i x}{2}\right)}{\Gamma\left(\frac{1}{2}+\frac{i x}{2}\right)}=-\frac{i x}{2}\left(\ln \frac{x^{2}}{4}-2\right)-\frac{i}{6 x}+\mathcal{O}\left(\frac{1}{x^{3}}\right) .
$$


Thus

$$
\begin{aligned}
2 \operatorname{Re}\left[\psi\left(\frac{1}{2}-\frac{i x}{2}\right)\right]= & 2 i \frac{d}{d x} \ln \frac{\Gamma\left(\frac{1}{2}-\frac{i x}{2}\right)}{\Gamma\left(\frac{1}{2}+\frac{i x}{2}\right)} \\
= & \ln \frac{x^{2}}{4}-\frac{1}{3 x^{2}}+\mathcal{O}\left(\frac{1}{x^{4}}\right) \\
2 \operatorname{Re}\left[\zeta^{\prime}\left(-1, \frac{1}{2}-\frac{i x}{2}\right)\right]= & -\frac{x^{2}}{8}\left(\ln \frac{x^{2}}{4}-1\right) \\
& -\frac{1}{24} \ln x^{2}+\mathcal{O}\left(x^{0}\right) .
\end{aligned}
$$

The last expansion can be derived with the help of Eq. (A17) after noting that the zeta function satisfies the relation

$$
\frac{\partial}{\partial q} \zeta^{\prime}(z, q)=-\zeta(z+1, q)-z \zeta^{\prime}(z+1, q)
$$

and that $\zeta(0, q)=1 / 2-q$. Low temperature expansion of the integrals A4, A5 and A13 is now straightforward. We get

$$
\begin{aligned}
I_{1}^{0} & =-\frac{\mu^{2}}{8 \pi^{2}}\left(1+\frac{\pi^{2}}{3} \frac{T^{2}}{\mu^{2}}+\epsilon\left[\left(1+\frac{\pi^{2}}{3} \frac{T^{2}}{\mu^{2}}\right) \ln \frac{\bar{\mu}^{2}}{\mu^{2}}+3-2 \ln 2+\mathcal{O}\left(\frac{T^{2}}{\mu^{2}}\right)\right]\right)+\mathcal{O}\left(\epsilon^{2}\right) \\
I_{2}^{0} & =\frac{1}{16 \pi^{2}}\left(\frac{1}{\epsilon}+\ln \frac{\bar{\mu}^{2}}{\mu^{2}}-2 \ln 2+\frac{\pi^{2}}{3} \frac{T^{2}}{\mu^{2}}+\mathcal{O}\left(\frac{T^{4}}{\mu^{4}}\right)\right)+\mathcal{O}(\epsilon) \\
I_{\text {sunset }} & =-\frac{\mu^{2}}{64 \pi^{4}}\left(\frac{1}{\epsilon}+\ln \frac{\bar{\mu}^{2}}{\mu^{2}}-4 \ln 2+6-\frac{2 \pi^{2}}{3} \frac{T^{2}}{\mu^{2}}+\mathcal{O}\left(\frac{T^{4}}{\mu^{4}}\right)\right)+\mathcal{O}(\epsilon) .
\end{aligned}
$$

Thus, although separate terms of the integrals diverge logarithmically at $T / \mu \rightarrow 0$, the integrals themselves are convergent in that limit, as they should.
[1] D. A. Kirzhnits, JETP Lett. 15 (1972) 529 [Pisma Zh. Eksp. Teor. Fiz. 15 (1972) 745]. D. A. Kirzhnits and A. D. Linde, Phys. Lett. B 42 (1972) 471.

[2] G. W. Anderson and L. J. Hall, Phys. Rev. D 45 (1992) 2685.

[3] M. E. Carrington, Phys. Rev. D 45 (1992) 2933.

[4] M. Dine, R. G. Leigh, P. Y. Huet, A. D. Linde and D. A. Linde, Phys. Rev. D 46 (1992) 550 arXiv:hep-ph/9203203.

[5] P. Arnold and O. Espinosa, Phys. Rev. D 47 (1993) $3546 \quad$ [Erratum-ibid. D $50 \quad$ (1994) 6662] arXiv:hep-ph/9212235, Z. Fodor and A. Hebecker, Nucl. Phys. B 432 (1994) 127 arXiv:hep-ph/9403219.

[6] K. Kajantie, M. Laine, K. Rummukainen and M. E. Shaposhnikov, Nucl. Phys. B 458 (1996) 90 arXiv:hep-ph/9508379.

[7] K. Kajantie, M. Laine, K. Rummukainen and M. E. Shaposhnikov, Nucl. Phys. B 466 (1996) 189 arXiv:hep-lat/9510020, Phys. Rev. Lett. 77 (1996) 2887 arXiv:hep-ph/9605288, Nucl. Phys. B 493 (1997) 413 arXiv:hep-lat/9612006, F. Karsch, T. Neuhaus, A. Patkos and J. Rank, Nucl. Phys. Proc. Suppl. 53 (1997) 623 arXiv:hep-lat/9608087, M. Gurtler, E. M. Ilgenfritz and A. Schiller, Phys. Rev. D 56 (1997) 3888 arXiv:hep-lat/9704013,

[8] F. Csikor, Z. Fodor and J. Heitger, Phys. Rev. Lett. 82 (1999) 21 arXiv:hep-ph/9809291.

[9] K. Rummukainen, M. Tsypin, K. Kajantie, M. Laine and M. E. Shaposhnikov, Nucl. Phys. B 532 (1998) 283 arXiv:hep-lat/9805013.
[10] K. Kajantie, M. Laine, J. Peisa, K. Rummukainen and M. E. Shaposhnikov, Nucl. Phys. B 544 (1999) 357 arXiv:hep-lat/9809004.

[11] M. Orito, T. Kajino, G. J. Mathews and Y. Wang, Phys. Rev. D 65 (2002) 123504 arXiv:astro-ph/0203352.

[12] B. Bajc, A. Riotto and G. Senjanovic, Phys. Rev. Lett. 81 (1998) 1355 arXiv:hep-ph/9710415, J. McDonald, Phys. Lett. B 463 (1999) 225 arXiv:hep-ph/9907358, B. Bajc and G. Senjanovic, Phys. Lett. B 472 (2000) 373 arXiv:hep-ph/9907552.

[13] J. McDonald, Phys. Rev. Lett. 84 (2000) 4798 arXiv:hep-ph/9908300, J. March-Russell, H. Murayama and A. Riotto, JHEP 9911 (1999) 015 arXiv:hep-ph/9908396.

[14] A. D. Linde, Phys. Rev. D 14 (1976) 3345.

[15] A. D. Linde, Phys. Lett. B 86 (1979) 39.

[16] D. Bailin and A. Love, Nucl. Phys. B 226 (1983) 493.

[17] E. J. Ferrer, V. de la Incera and A. E. Shabad, Phys. Lett. B 185 (1987) 407.

[18] J. I. Kapusta, Phys. Rev. D 42 (1990) 919.

[19] S. Y. Khlebnikov and M. E. Shaposhnikov, Phys. Lett. B 387 (1996) 817 arXiv:hep-ph/9607386.

[20] M. Laine and M. E. Shaposhnikov, Phys. Rev. D 61 (2000) 117302 arXiv:hep-ph/9911473.

[21] F. Sannino, arXiv:hep-ph/0211367 F. Sannino and W. Schafer, Phys. Lett. B $\mathbf{5 2 7}$ (2002) 142 arXiv:hep-ph/0111098.

[22] V. A. Rubakov and M. E. Shaposhnikov, Usp. Fiz. Nauk 166 (1996) 493 [Phys. Usp. 39 (1996) 461] arXiv:hep-ph/9603208. 
[23] P. Ginsparg, Nucl. Phys. B 170 (1980) 388, T. Appelquist and R. D. Pisarski, Phys. Rev. D 23 (1981) 2305.

[24] G. V. Dunne, arXiv:hep-th/9902115

[25] A. N. Redlich and L. C. Wijewardhana, Phys. Rev. Lett. 54 (1985) 970.

[26] V. A. Rubakov and A. N. Tavkhelidze, Phys. Lett. B 165 (1985) 109, V. A. Rubakov, Prog. Theor. Phys. 75 (1986) 366, D. V. Deryagin, D. Y. Grigoriev and V. A. Rubakov,
Phys. Lett. B 178 (1986) 385.

[27] A. Vuorinen, Phys. Rev. D $67 \quad$ (2003) 074032 arXiv:hep-ph/0212283.

[28] P. Arnold and C. X. Zhai, Phys. Rev. D 50 (1994) 7603 arXiv:hep-ph/9408276. Phys. Rev. D 51 (1995) 1906 arXiv:hep-ph/9410360. 